\title{
SOME CANONICAL BIFURCATIONS IN THE SWIFT-HOHENBERG EQUATION
}

\author{
L.A. PELETIER AND J.F. WILLIAMS
}

\begin{abstract}
We study stationary solutions $u(x)$ and their stability of the fourth order Swift-Hohenberg equation on a bounded domain $(0, L)$ with boundary conditions $u=0$ and $u^{\prime \prime}=0$ at $x=0$ and $x=L$. It is well known that as $L$ increases the set of stationary solutions becomes increasingly complex. Numerical studies have have exhibited two interesting types of structures in the bifurcation diagram for $(L, u)$. In this paper we demonstrate through a center manifold analysis how these structures arise naturally near certain bifurcation points, and that there are no others. We also analyze their stability properties.
\end{abstract}

\section{INTRODUCTION}

In this paper we consider solutions of the Swift-Hohenberg equation

$$
\frac{\partial u}{\partial t}=\alpha u-\left(1+\frac{\partial^{2}}{\partial x^{2}}\right)^{2} u-u^{3}, \quad \alpha \in \mathbf{R},
$$

on the cylindrical domain $Q=(0, L) \times \mathbf{R}^{+}$subject to the boundary conditions

$$
u=0 \quad \text { and } \quad \frac{\partial^{2} u}{\partial x^{2}}=0 \quad \text { at } \quad x=0, L .
$$

It is well known (see [1], [3], and Chapter 9 in the monograph [19]) that equation (1.1) has an increasing number of stationary solutions which satisfy the boundary conditions (1.2) as the length $L$ of the domain increases. The objective of this paper is to study the set of stationary solutions as it depends on $\alpha$ and $L$. In particular, we focus on understanding certain complex but recurrent structures in the bifurcation diagram which were first observed in numerical studies

In studies of pattern formation, the Swift-Hohenberg equation plays a central role. Proposed in 1977 by Swift \& Hohenberg [21] in connection with Rayleigh-Bénard convection, it has since featured in a variety of problems, such as Taylor-Couette flow [14], [16], and in the study of lasers [15]. For further references we mention the surveys given in [8] and [9] and the recent review [2].

We view the Swift-Hohenberg equation as a model equation for a large class of higher order parabolic model equations arising in a wide range of applications, such as the Extended Fisher-Kolmogorov equation in statistical mechanics [10], [22], and a sixth order equation introduced by Caginalp and Fife in phase field models [4],

Date: December 15, 2005.

Research supported by RTN network HPRN-CT-2002-00274. 
[11]. Many of the phenomena observed here for the Swift-Hohenberg equation are found in the dynamics of such equations as well [3], [5], [6], [7] and [19].

The Swift-Hohenberg equation is interesting from the point of view of pattern formation, because of its many qualitatively different stable equilibrium solutions. This begs the question, which of these equilibrium solutions will be selected as time tends to infinity, and how this selection depends on the parameters $\alpha$ and $L$ involved in the problem.

We study the Cauchy-Dirichlet problem for the Swift-Hohenberg equation on the cylinder $Q=(0, L) \times \mathbf{R}^{+}$with boundary conditions (1.2). Thus, writing equation (1.1) in a more conventional form, we consider the problem

$$
\left\{\begin{array}{lr}
u_{t}=-u_{x x x x}-2 u_{x x}-(1-\alpha) u-f(u) & \text { for } 0<x<L, t>0 \\
u=0 \text { and } u_{x x}=0 & \text { at } \quad x=0, L, t>0, \\
u(x, 0)=u_{0}(x) & \text { for } 0<x<L,
\end{array}\right.
$$

where we consider nonlinearities $f(u)$ which are either stabilizing or de-stabilizing. We shall assume that $f$ is a smooth function, and that $f(s)=o(s)$ as $s \rightarrow 0$. As typical examples of such functions we choose

$$
f(s)=s^{3} \text { (stabilizing) } \quad \text { or } \quad f(s)=-s^{3}+s^{5} \text { (de-stabilizing). }
$$

The boundary conditions have been chosen so that solutions can be extended as periodic functions on $\mathbf{R}$. The initial function $u_{0}$ is a smooth function that vanishes at $x=0$ and $x=L$. In most of this article we will assume that $u_{0}$ is symmetric with respect to the center of the domain, i.e.

$$
u_{0}(L-x)=u_{0}(x) \quad \text { for } \quad 0<x<L .
$$

Note that this assumption implies that the solution remains symmetric for all $t$. It is motivated by the fact that it increases the set of lengths $L$ for which the trivial solution is globally stable [17],[18].

Problem (1.3) is a Gradient System with corresponding Lyapunov functional

$$
J(u ; L)=\frac{1}{L} \int_{0}^{L}\left\{\frac{1}{2}\left(u^{\prime \prime}\right)^{2}-\left(u^{\prime}\right)^{2}+\frac{1-\alpha}{2} u^{2}+F(u)\right\} d x
$$

where $F^{\prime}(s)=f(s)$ and $F(0)=0$. This means (cf. [13]) that if the stationary solutions of Problem (1.3) are isolated, then $u(x, t)$ tends to one of these solutions as $t \rightarrow \infty$. i.e., for every $x \in(0, L)$ :

$$
u(x, t) \rightarrow v(x) \quad \text { as } \quad t \rightarrow \infty,
$$

where $v(x)$ is a solution of the two-point boundary value problem

$$
\begin{cases}v^{i v}+2 v^{\prime \prime}+(1-\alpha) v+f(v)=0 & \text { for } \quad 0<x<L, \\ v=0 \text { and } v^{\prime \prime}=0 & \text { at } \quad x=0, L .\end{cases}
$$

For the stabilizing case it is known that if $\alpha \leq 0$, then Problem (1.3) has the trivial solution only (cf. Chapter 9 of [19]), and hence, for every $x \in(0, L)$,

$$
u(x, t) \rightarrow 0 \quad \text { as } \quad t \rightarrow \infty .
$$



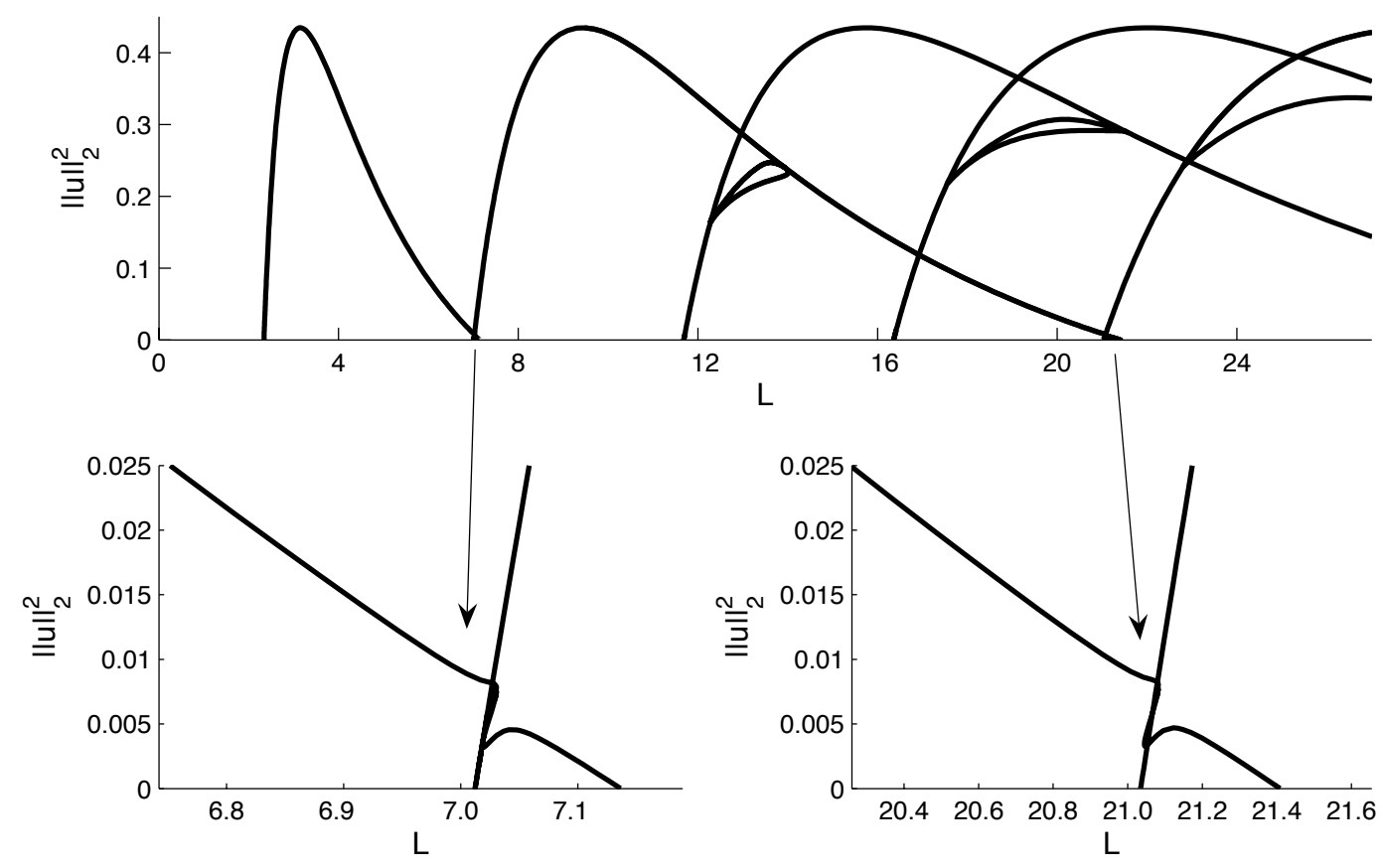

Figure 1. Global bifurcation diagram when $\alpha=0.65$

For both cases the situation is much more complex when $\alpha>0$ and, depending on the value of $\alpha$ and $L$, there may be very many stationary profiles to choose from. In this article we make a start with answering the question, which of these profiles is chosen.

In [17], [18] a series of numerical simulations was carried out on the SwiftHohenberg equation in order to gain insight into the different types of limiting behaviors and the effect of the parameters $\alpha$ and $L$ on the final profile that is selected. One of the striking observations was that for values of $\alpha$ smaller than, say, $\frac{1}{4}$ critical lengths $L_{1}$ and $L_{2}$ could be identified such that the solution converged to one type of limit for $L_{1}<L<L_{2}$, and to another when $3 L_{1}<L<3 L_{2}$, provided the intervals $\left[L_{1}, L_{2}\right],\left[3 L_{1}, 3 L_{2}\right]$ and $\left[5 L_{1}, 5 L_{2}\right]$ are disjunct. In between, for $L \in\left[L_{2}, 3 L_{1}\right]$ and for $L \in\left[3 L_{2}, 5 L_{1}\right]$ the trivial solution proved globally stable.

When the above intervals overlap, the simulations were not very revealing. This motivated us to carry out a numerical study of solution branches of the stationary problem (1.8). In Figure 1 we present a result of such a study for $\alpha=0.65$. It reveals interesting qualitatively new structures in the intersections of the intervals, which are quite robust under changes of $\alpha$. We see that loop-type branches bifurcate from the trivial solution at $L=n L_{1}$ and $L=n L_{2}, n=1,3,5, \ldots$. But in addition we see 

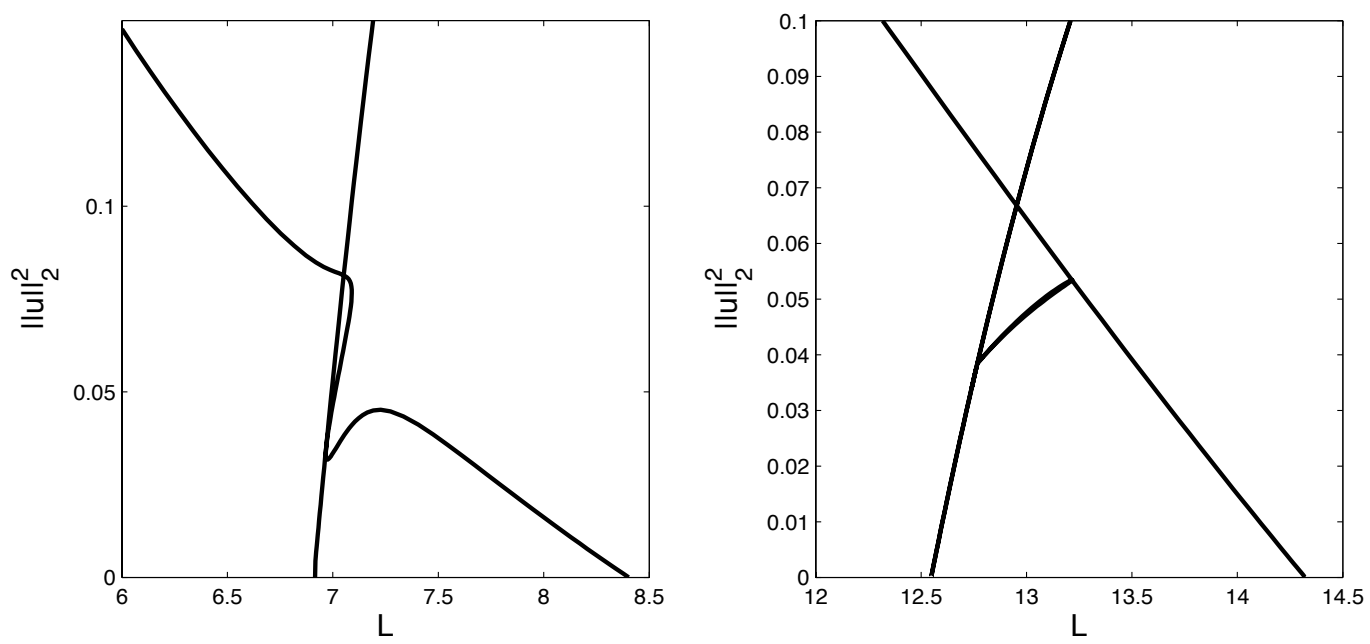

FiguRe 2. Detail of bifurcation diagrams when $\left[L_{1}, L_{2}\right]$ and $\left[3 L_{1}, 3 L_{2}\right]$ overlap (left) and when $\left[3 L_{1}, 3 L_{2}\right]$ and $\left[5 L_{1}, 5 L_{2}\right]$ overlap (right).

(A) Comparable - nontrivial - structures in the intersection of the intervals $\left[L_{1}, L_{2}\right]$ and $\left[3 L_{1}, 3 L_{2}\right]$, and in the intersection of the intervals $\left[3 L_{1}, 3 L_{2}\right]$ and $\left[9 L_{1}, 9 L_{2}\right]$.

(B) Pairs of branches which connect the loop-type branch bifurcating from $5 L_{1}$ to the one from $3 L_{1}$ and to the one from $7 L_{1}$.

In this paper we analyze these structures by means of a center manifold analysis carried out when, for instance, $3 L_{1} \approx L_{2}$ and the intervals $\left[L_{1}, L_{2}\right]$ and $\left[3 L_{1}, 3 L_{2}\right]$ slightly overlap.

We shall show that when $n L_{1} \approx m L_{2}$ and the intervals $\left(m L_{1}, m L_{2}\right)$ and $\left(n L_{1}, n L_{2}\right)$ slightly ovelap for certain odd integers $m$ and $n$, then two types of structures may be distinguished:

(i) When $n=3 m$ we obtain the structure shown in Figure 2 (left), and

(ii) When $n \neq 3 m$ we obtain the structure shown in Figure 2 (right)

and these are the only two possible structures. In addition, only if $m=1$ and $n=3$ is the dimension of the unstable manifold $X^{u}$ equal to zero. In all other cases it is positive.

The plan of the paper is the following. First, in Section 2, we recall some results about the stability properties of the linearized Swift-Hohenberg equation. Then, in Section 3 we discuss stationary solutions in the limit as $\alpha \rightarrow 0$. In Section 4 , we turn to the first characteristic solution set when $3 L_{1} \approx L_{2}$ and in Section 5 , we generalize the analysis to the situation when $n L_{1} \approx m L_{2}$. In Section 6 we compute the Lyapunov functions for the solutions found in Sections 4 and 5, and inspect, which of the solutions is the global minimizer, given a value of $L$. Finally, in Section 7 , we apply the techniques used in the previous sections to the Swift-Hohenberg equation with the de-stabilizing quintic nonlinearity: $f(s)=-s^{3}+s^{5}$. 


\section{The Linearized Swift-Hohenberg Equation}

In this section we recall some properties of the linear problem associated with Problem (1.3):

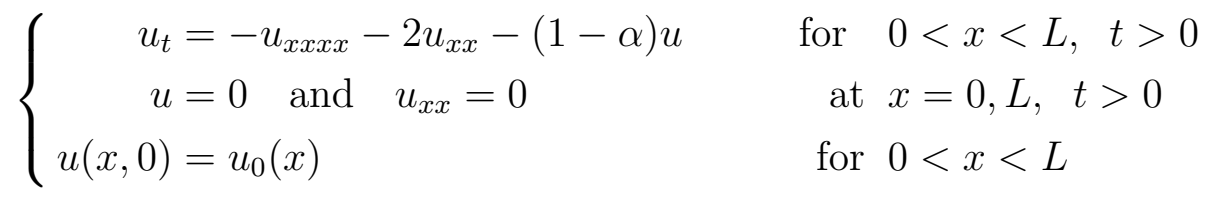

The stability of the zero solution to (2.1) is determined by examining the Ansatz $u(x, t)=\varphi(x) e^{-\lambda t}$. This leads to the eigenvalue problem

$$
\begin{cases}\varphi^{(i v)}+2 \varphi^{\prime \prime}+(1-\alpha) \varphi=\lambda \varphi & \text { for } 0<x<L, \\ \varphi=0 \text { and } \varphi^{\prime \prime}=0 & \text { at } x=0, L .\end{cases}
$$

The eigenvalues $\lambda_{n}$ and the eigenfunctions $\varphi_{n}$ are given by

$$
\lambda_{n}=\lambda_{n}(L)=P\left(\frac{n \pi}{L}\right) \quad \text { and } \quad \varphi_{n}(x)=\sqrt{2} \sin \left(\frac{n \pi x}{L}\right),
$$

where $P(\xi)$ is the symbol of the operator on the left hand side of $(2.2)$ :

$$
P(\xi)=\left(\xi^{2}-1\right)^{2}-\alpha .
$$

and the eigenfunctions have been normalized that

$$
\left(\varphi_{m}, \varphi_{n}\right) \stackrel{\text { def }}{=} \frac{1}{L} \int_{0}^{L} \varphi_{m}(x) \varphi_{n}(x) d x=1 \quad \text { if } \quad m=n .
$$

What is interesting about this equation is that given $\alpha \in(0,1)$ is small enough, there are domain lengths for which the zero solution is stable and others for which it is not. Let $\alpha \in(0,1)$. Then $P(\xi)$ has two zeros $\xi_{ \pm}\left(0<\xi_{-}<\xi_{+}\right)$, so that $P<0$ on $\left(\xi_{-}, \xi_{+}\right)$and $P>0$ on $\left(0 . \xi_{-}\right) \cup\left(\xi_{+}, \infty\right)$. This implies that

$$
\lambda_{n}(L)<0 \quad \text { when } \quad L \in\left(n L_{1}, n L_{2}\right)
$$

where $L_{1}=\pi / \xi_{+}$and $L_{2}=\pi / \xi_{-}$, i.e.,

$$
L_{1}=\frac{\pi}{\sqrt{1+\sqrt{\alpha}}} \quad \text { and } \quad L_{2}=\frac{\pi}{\sqrt{1-\sqrt{\alpha}}}
$$

This phenomenon is exhibited in Figure 3. Here we have solved the CauchyDirichlet Problem (1.3) numerically for $\alpha=1 / 4$ and $\alpha=5 / 8$, and randomly chosen initial data. The $L^{2}$-norm of the limiting solution $v(x)$ is plotted against the domain length clearly showing regions when the zero solution is or is not stable. Details of the numerical simulations are discussed in Appendix B.

In order the analyze the solution branches on the $(L, u)$-plane, we vary $\alpha$ so that two critical domain lengths coalesce:

$$
\text { Case I: } L_{2}-L_{1} \searrow 0 \text { and Case II: } L_{2}-3 L_{1} \searrow 0 \text {. }
$$

The first case arises when $\alpha \rightarrow 0$. Here we will formally construct the solutions in this limit to describe the branch of solutions connecting $L_{1}$ and $L_{2}$ as depicted in Figure 3. 

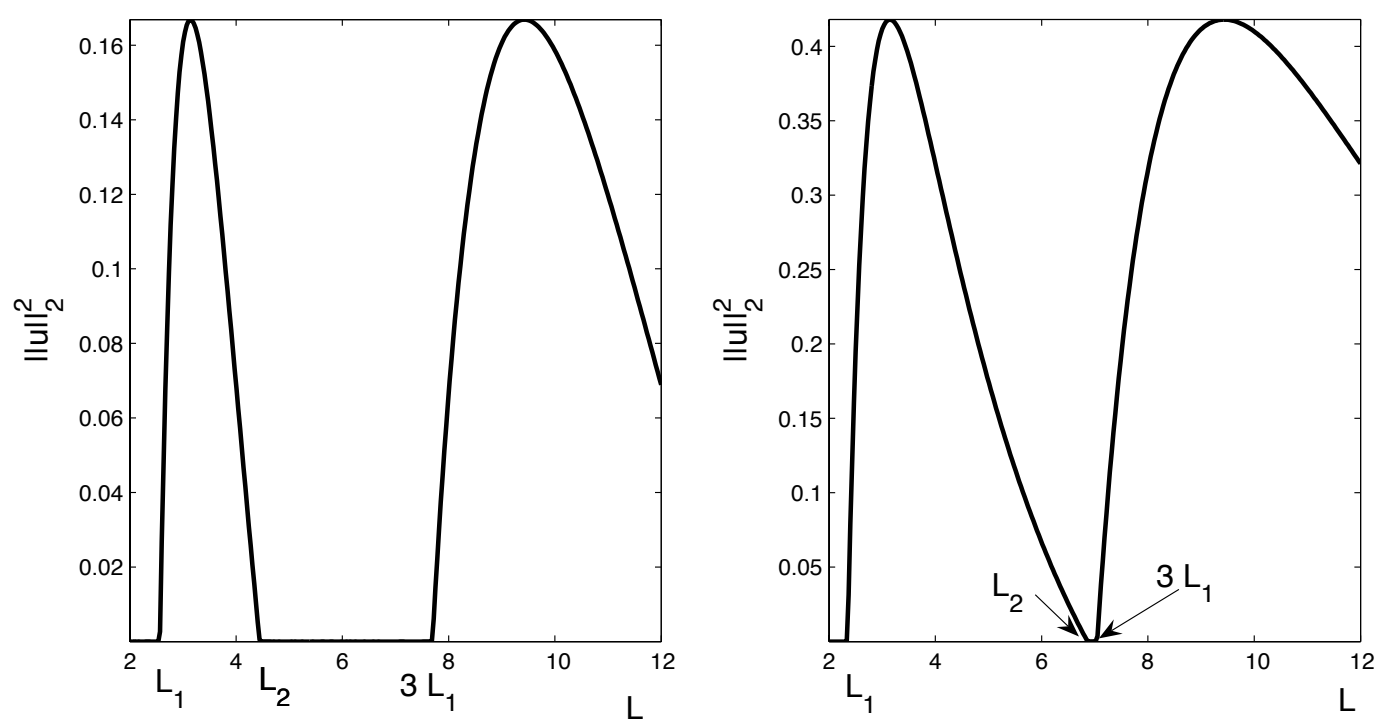

FiguRe 3. $L^{2}$-norms of stable solutions as a function of $L$. In the left figure $\alpha=1 / 4$ while in the right $\alpha=5 / 8$. Notice that as $\alpha$ increases further, the two solution branches come together at the base line. They meet when $\alpha=\alpha_{1}^{*}=16 / 25$.

The second case arises when

$$
\alpha \searrow \alpha_{1}^{*} \stackrel{\text { def }}{=} \frac{16}{25} \quad \text { and } \quad L_{1}^{*}=3 L_{1}\left(\alpha_{1}^{*}\right)=L_{2}\left(\alpha_{1}^{*}\right)=\pi \sqrt{5} .
$$

In [17], [18] the local shape near $L=L_{1}$ and $L=L_{2}$ of this diagram was determined. Specifically, the following results were established:

Theorem 2.1. Let $\alpha>0$. There exists a unique branch in the $(L, u)$-plane of nontrivial solutions of Problem (1.3) which emanates from the trivial solution at $L_{1}$. Its local behavior is given by

$$
\|u\|^{2} \sim \frac{8}{3 \pi} \sqrt{\alpha}(1+\sqrt{\alpha})^{3 / 2}\left(L-L_{1}\right) \quad \text { as } \quad L \searrow L_{1} .
$$

Theorem 2.2. Let $0<\alpha<1$. There exists a unique branch in the $(L, u)$-plane of nontrivial solutions of Problem (1.3) which emanates from the trivial solution at $L_{2}$. Its local behavior is given by

$$
\|u\|^{2} \sim \frac{8}{3 \pi} \sqrt{\alpha}(1-\sqrt{\alpha})^{3 / 2}\left(L_{2}-L\right) \quad \text { as } \quad L \nearrow L_{2} .
$$

Here $\|u\|^{2}=(1 / L) \int_{0}^{L} u^{2}(x) d x$.

It is our present interest to understand the full time-dependent problem near those values of $\alpha$ and $L$ where these bifurcation points approximately coalesce.

We have the following center manifold theorem: 
Theorem 2.3. Suppose that $\alpha=\alpha_{1}^{*}$ and so $3 L_{1}=L_{2}$. Then if $L=3 L_{1}$,

(a) Problem (1.3) has a two-dimensional center manifold $X^{c}$ about the trivial solution which is spanned by $\varphi_{1}$ and $\varphi_{3}$.

(b) The dimension of the unstable manifold $X^{u}$ is zero.

Proof. If we extend Problem (1.3) to include $\alpha_{t}=0$, and linearize about $u(x, t)=0$ and $\alpha=\alpha_{1}^{*}$, we obtain the system

$$
\left\{\begin{aligned}
u_{t} & =-u_{x x x x}-2 u_{x x}-(1-\alpha) u & & \text { for } 0<x<L, t>0 \\
u & =0 \text { and } u_{x x}=0 & & \text { at } x=0, L, t>0 \\
\alpha_{t} & =0 & & \text { for } t>0 .
\end{aligned}\right.
$$

The eigenfunctions and eigenvalues of Problem (2.4) are given by (2.3). Since $\alpha$ has been chosen such that $3 L_{1}=L_{2}$, and $L=3 L_{1}$, it follows that

$$
\lambda_{1}(L)=0 \quad \text { and } \quad \lambda_{3}(L)=0 .
$$

A simple calculation shows that

$$
\lambda_{n}=\left(\left(\frac{n}{\sqrt{5}}\right)^{2}-1\right)^{2}-\frac{16}{25}=\frac{n^{2}}{5}\left(\frac{n^{2}}{25}-2\right)+\frac{9}{25}>0 \quad \text { for } n=5,7, \ldots .
$$

so that all the eigenvalues, starting from $\lambda_{5}$, are positive. Therefore, $(i)$ there exists a two-dimensional center manifold for this value $\alpha=\alpha_{1}^{*}$ which is spanned by $\varphi_{1}$ and $\varphi_{3}$, and $(i i)$ the unstable manifold has dimension zero.

By smoothness this manifold persists for small changes in $\alpha$ and $L$ [20].

\section{Stationary SOLUTiOns in The Limit AS $\alpha \rightarrow 0^{+}$}

In this section we construct asymptotic expressions for nontrivial stationary solutions of Problem (1.3) in the limit as $\alpha \rightarrow 0^{+}$when the nonlinearity is cubic, i.e. when it is given by $f(s)=s^{3}$. Thus, we seek to describe nontrivial solutions of the problem

$$
\left\{\begin{array}{lr}
v^{i v}+2 v^{\prime \prime}+(1-\alpha) v+v^{3}=0 \quad \text { for } & 0<x<L, \\
v=0 \text { and } v^{\prime \prime}=0 & \text { at } \quad x=0, L .
\end{array}\right.
$$

We choose $L \in\left(n L_{1}, n L_{2}\right)$, where $n$ is an arbitrary positive integer, so that a nontrivial solution is known to exist, and we choose $\alpha$ so small that the intervals $\left[n L_{1}, n L_{2}\right]$ and $\left[(n+2) L_{1},(n+2) L_{2}\right]$ are disjoint. This is the case when $\alpha<\alpha_{n}^{*}$, where

$$
\alpha_{n}^{*}=\frac{4(n+1)^{2}}{\left(n^{2}+2 n+2\right)^{2}} \text {. }
$$

It will be convenient to rescale the spatial variable so that the domain $(0, L)$ maps onto the fixed domain $(0,1)$. Thus, we put

$$
y=L x \quad \text { and } \quad w(y)=v(x) .
$$


We then find that Problem (3.1) becomes

$$
\left\{\begin{array}{lrl}
w^{i v}+2 L^{2} w^{\prime \prime}+L^{4}\left((1-\alpha) w+w^{3}\right)=0, & \text { for } \quad 0<x<1 \\
v=0, \quad v^{\prime \prime}=0 & \text { at } \quad x=0,1 .
\end{array}\right.
$$

We are interested in the situation that $\alpha \rightarrow 0^{+}$. The existence of branches bifurcating from $L_{1}$ and $L_{2}$ is established in Theorems 2.1 and 2.2. These local results show that as $L \rightarrow L_{1}^{+}$and $L \rightarrow L_{2}^{-}$the solution scales like $\sqrt{\alpha}$. We therefore seek an expansion of $w(y)$ in a series of the form

$$
w(y) \sim \alpha^{1 / 2} w_{1}(y)+\alpha w_{2}(y)+\alpha^{3 / 2} w_{3}(y)+\alpha^{2} w_{4}(y)+\ldots
$$

We introduce a scaling factor $\delta \in[0,1]$ and write

$$
L=n \pi L_{1}+n \pi \delta\left(L_{2}-L_{1}\right)=n \pi\left\{L_{1}+\delta L_{\text {gap }}\right\}, \quad 0 \leq \delta \leq 1
$$

and expand $L$ in the limit as $\alpha \rightarrow 0^{+}$. A simple computation shows that

$$
L_{1}(\alpha)=\frac{\pi}{\sqrt{1+\sqrt{\alpha}}}=\pi\left(1-\frac{1}{2} \alpha^{1 / 2}+\frac{3}{8} \alpha-\frac{5}{16} \alpha^{3 / 2}+\ldots\right)
$$

and

$$
L_{2}(\alpha)=\frac{\pi}{\sqrt{1-\sqrt{\alpha}}}=\pi\left(1+\frac{1}{2} \alpha^{1 / 2}+\frac{3}{8} \alpha+\frac{5}{16} \alpha^{3 / 2}+\ldots\right)
$$

so that

$$
L_{\text {gap }}(\alpha) \stackrel{\text { def }}{=} L_{2}(\alpha)-L_{1}(\alpha) \sim \pi\left(\alpha^{1 / 2}+\frac{5}{8} \alpha^{3 / 2}+\ldots\right) \quad \text { as } \quad \alpha \rightarrow 0^{+} .
$$

Therefore as $\alpha \rightarrow 0^{+}$we have

$$
L(\alpha)=n \pi\left\{1+\frac{1}{2}(2 \delta-1) \alpha^{1 / 2}+\frac{3}{8} \alpha+\frac{5}{16}(2 \delta-1) \alpha^{3 / 2}+\ldots\right\} .
$$

We now expand (3.2), using (3.3) and (3.4), in terms of powers of $\alpha^{1 / 2}$ and equate the coefficients of $\alpha^{1 / 2}, \alpha, \alpha^{3 / 2}$ and $\alpha^{2}$ equal to zero. We then obtain the equations

$$
\begin{aligned}
\mathcal{O}\left(\alpha^{1 / 2}\right): & \mathcal{L}_{1} w_{1}=0, \\
\mathcal{O}(\alpha): & \mathcal{L}_{1} w_{2}+\mathcal{L}_{2} w_{1}=0, \\
\mathcal{O}\left(\alpha^{3 / 2}\right): & \mathcal{L}_{1} w_{3}+\mathcal{L}_{2} w_{2}+\mathcal{L}_{3}\left(w_{1}\right)+n^{4} \pi^{4} w_{1}^{3}=0, \\
\mathcal{O}\left(\alpha^{2}\right): & \mathcal{L}_{1} w_{4}+\mathcal{L}_{2} w_{3}+\mathcal{L}_{3} w_{2}+\mathcal{L}_{4} w_{1}+n^{4} \pi^{4}\left\{3 w_{1}^{2} w_{2}+2(2 \delta-1) w_{1}^{3}\right\}=0,
\end{aligned}
$$

where

$$
\begin{aligned}
& \mathcal{L}_{1} z \stackrel{\text { def }}{=} z^{i v}+2 n^{2} \pi^{2} z^{\prime \prime}+n^{4} \pi^{4} z \\
& \mathcal{L}_{2} z \stackrel{\text { def }}{=} 2 n^{2} \pi^{2}(2 \delta-1)\left(z^{\prime \prime}+n^{2} \pi^{2} z\right) \\
& \mathcal{L}_{3} z \stackrel{\text { def }}{=} 2 n^{2} \pi^{2}\{1-\delta(1-\delta)\} z^{\prime \prime}+n^{4} \pi^{4}\{2-6 \delta(1-\delta)\} z, \\
& \mathcal{L}_{4} z \stackrel{\text { def }}{=} 2 n^{2} \pi^{2}(2 \delta-1)\left(z^{\prime \prime}+n^{2} \pi^{2}\{2-\delta(1-\delta)\} z\right) .
\end{aligned}
$$


The functions $w_{j}(y), j=1,2,3,4, \ldots$ all need to satisfy the boundary conditions of Problem (3.2):

$$
w_{j}(y)=0 \quad \text { and } \quad w_{j}^{\prime \prime}(y)=0 \quad \text { at } \quad y=0,1 .
$$

This means that

$$
w_{1}(y)=\gamma_{1} \sin (n \pi y)
$$

where $\gamma_{1}$ still needs to be determined. Since $\mathcal{L}_{2} w_{1}=0$, the equation for $w_{2}$ reduces to $\mathcal{L}_{1} w_{2}=0$, so that

$$
w_{2}(y)=\gamma_{2} \sin (n \pi y)
$$

as well, and $\gamma_{2}$ must also be determined. To fix $\gamma_{1}$ we enforce the solvability condition for the equation for $w_{3}$ :

$$
\left(w_{1}, \mathcal{L}_{1} w_{3}\right)=-\left(w_{1}, \mathcal{L}_{2} w_{2}+\mathcal{L}_{3} w_{1}+n^{4} \pi^{4} w_{1}^{3}\right)=0
$$

where $(\cdot, \cdot)$ is the standard inner product in $L^{2}(0,1)$. Since $\mathcal{L}_{2}$ is self adjoint, and $\mathcal{L}_{2} w_{1}=0$, this condition reduces to

$$
\left(w_{1}, \mathcal{L}_{3} w_{1}+n^{4} \pi^{4} w_{1}^{3}\right)=0
$$

which yields

$$
\gamma_{1}(\delta)=\frac{4}{\sqrt{3}} \sqrt{\delta(1-\delta)} \quad \text { with } \quad \delta \in[0,1] .
$$

To determine $\gamma_{2}$ we use the solvability condition for the problem for $w_{4}$ term in the expansion. This yields the condition

$$
\left(w_{1}, \mathcal{L}_{3} w_{2}+\mathcal{L}_{4} w_{1}+n^{4} \pi^{4}\left\{3 w_{1}^{2} w_{2}+2(2 \delta-1) w_{1}^{3}\right\}\right)=0,
$$

from which we derive $\gamma_{2}$. The first two terms of the expansion are thus found to be:

$$
w(y ; \delta)=\sqrt{\delta(1-\delta)}\left(\frac{4}{\sqrt{3}} \sqrt{\alpha}+\alpha \sqrt{3}(1-2 \delta)\right) \sin (n \pi y)+\ldots
$$

A comparison of the branch predicted by this formula against numerical computations is shown in Figure 4.

The stability of the solutions on this branch is easily computed by setting

$$
u(x, t) \simeq c(t) \sqrt{\alpha} v_{1}(x)+\ldots, \quad v_{1}(x)=\gamma_{1} \sin \left(\frac{n \pi x}{L}\right) .
$$

Clearly one asympotic solution of the full time-dependent PDE is $c(t)=\gamma_{1}$. Projecting this solution onto the local center manifold $X^{c}=\operatorname{span}\{\sin (n \pi x / L)\}$ and expanding to leading order in $\alpha$ leads to

$$
\dot{c}=-\frac{1}{8} c\left\{3 c^{2}-16 \delta(1-\delta)\right\} .
$$

Linearizing this equation about $c(t)=\gamma_{1}+d(t)$ for small $d$ yields

$$
\dot{d}=-4 \delta(1-\delta) d
$$

from which we conclude that this solution is stable for admissible $\delta$. This comes as no surprise as it is precisely in this interval that the zero solution is unstable.

The construction described above involves an interval $\left(n L_{1}, n L_{2}\right)$ which is disjunct from $\left((n+2) L_{1},(n+2) L_{2}\right)$. In Sections 4 and 5 we consider what happens when 

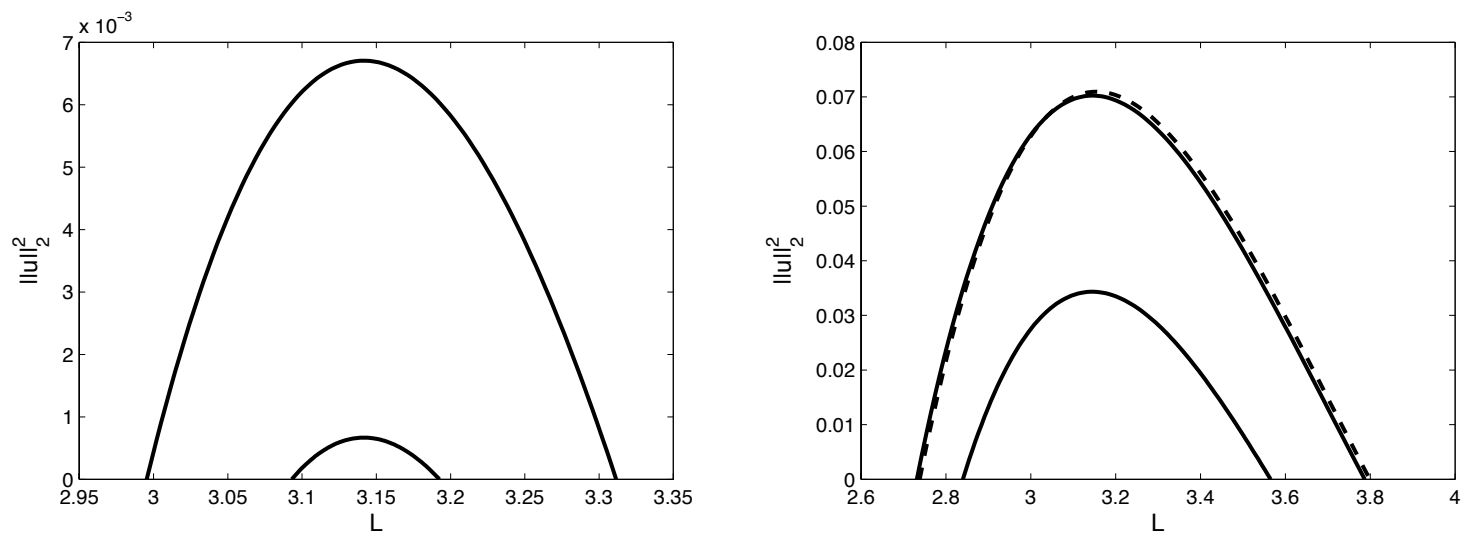

Figure 4. Comparison of the numerical and asymptotic structures of the branches for $\alpha=10^{-3}, 10^{-2}$ on the left and $510^{-2}$ and $10^{-1}$ on the right.

these two intervals overlap, albeit slightly, and we investigate the dynamics on a two-dimensional center manifold.

\section{Stationary solutions in the limit as $\alpha \rightarrow \alpha_{1}^{*}$.}

In this section we focus on the situation when the branch which connects the branchpoints at $L_{1}$ and $L_{2}$ overlaps with the branch which connects $3 L_{1}$ and $3 L_{2}$. As we saw in the Introduction, this will be the case when $\alpha>\alpha_{1}^{*}$. Numerical studies suggest that the set of stationary solutions then can become quite complex. In this section we analyse this set when the bifurcation points at $L_{2}$ and $3 L_{1}$ are close together, i.e. when $\alpha=\alpha_{1}^{*}+\varepsilon$ and $\varepsilon$ is positive and small.

As we have seen in Theorem 2.3 when $\alpha=\alpha_{1}^{*}$, then Problem (1.7) has a two dimensional center manifold $X^{c}$ about the trivial solution:

$$
X^{c}=\operatorname{span}\left\{\varphi_{1}, \varphi_{3}\right\}=\{\sin (x \pi / L), \sin (3 x \pi / L)\} .
$$

In our analysis we shall fix $\alpha$ close to $\alpha_{1}^{*}$ and use $L$ as a bifurcation parameter taking values close to $L_{1}^{*}=L_{2}\left(\alpha_{1}^{*}\right)$.

To determine the dynamics on the space $X^{c}$ we write

$$
u(x, t)=a(t) \varphi_{1}(x)+b(t) \varphi_{3}(x)
$$

and project the differential equation onto $X^{c}$ :

$$
\begin{aligned}
& \frac{1}{L} \int_{0}^{L}\left\{u_{t}+u_{x x x x}+2 u_{x x}+(1-\alpha) u+u^{3}\right\} \varphi_{1} d x=0 \\
& \frac{1}{L} \int_{0}^{L}\left\{u_{t}+u_{x x x x}+2 u_{x x}+(1-\alpha) u+u^{3}\right\} \varphi_{3} d x=0 .
\end{aligned}
$$


This yields a pair of differential equations for $a(t)$ and $b(t)$ :

$$
\left\{\begin{array}{l}
\dot{a}=-P\left(\frac{\pi}{L}\right) a-\frac{3}{2} a^{3}+\frac{3}{2} a^{2} b-3 a b^{2}, \\
\dot{b}=-P\left(\frac{3 \pi}{L}\right) b+\frac{1}{2} a^{3}-3 a^{2} b-\frac{3}{2} b^{3} .
\end{array}\right.
$$

where dots denote differentiation with respect to $t$.

Because the original equation is a gradient system, the reduced system is one as well. The corresponding Lyapunov functional (the projection of (1.6) onto $X^{c}$ ) is given by

$$
V(a, b)=\frac{1}{2} P\left(\frac{\pi}{L}\right) a^{2}+\frac{1}{2} P\left(\frac{3 \pi}{L}\right) b^{2}+\frac{3}{8} a^{4}-\frac{1}{2} a^{3} b+\frac{3}{2} a^{2} b^{2}+\frac{3}{8} b^{4} .
$$

Plainly,

$$
\dot{a}=-V_{a}(a, b) \quad \text { and } \quad \dot{b}=-V_{b}(a, b),
$$

where subscripts denote partial differentiation, and

$$
\frac{d V}{d t}=V_{a} \dot{a}+V_{b} \dot{b}=-\left(V_{a}^{2}+V_{b}^{2}\right) \leq 0 .
$$

Thus, the function $V(a, b)$ decreases along orbits.

We will consider the structure and stability of the set of stationary solutions of the system (4.1). They are defined by the pair of equations

$$
V_{a}(a, b)=0 \quad \text { and } \quad V_{b}(a, b)=0 .
$$

This solution set contains at least three distinct branches: $(i)$ the trivial state, $(i i)$ a branch bifurcating from the bifurcation point at $3 L_{1}$ and (iii) a branch emanating from $L_{2}$. The existence of these branches was established in [18], but there the structure was only described in the limit as $\|u\| \rightarrow 0$. However, because of the center manifold structure near $L_{1}^{*}=3 L_{1}=L_{2}$ at $\alpha_{1}^{*}$, we see that these branches persist under small changes in $\alpha$ and $L$ near the first bifurcation point $(u, L)=\left(0, L_{1}^{*}\right)$. It is their local structure and stability that we now wish to investigate.

We fix

$$
\alpha=\alpha_{1}^{*}+\varepsilon, \quad \varepsilon>0 .
$$

Then $3 L_{1}<L_{2}$ and we write $L$, which serves as a bifurcation parameter, as

$$
L=3 L_{1}+\delta L_{\text {gap }}, \quad \text { where } \quad L_{\text {gap }}=L_{2}-3 L_{1}, \quad \delta \in \mathbf{R} .
$$

Note that $L_{\text {gap }}>0$ since $3 L_{1}<L_{2}$ in this range of $\alpha$. The parameter $\delta$ positions the length $L$ with respect to the interval $\left(3 L_{1}, L_{2}\right)$ so that $\delta=0$ corresponds with $3 L_{1}$ and $\delta=1$ corresponds with $L_{2}$. In what follows we shall describe the set of stationary solutions of $(4.1)$ in the $(\delta, u)$-space, where $u=(a, b)$, and discuss their stability properties.

Plainly, $L_{\text {gap }}(\varepsilon) \rightarrow 0$ as $\varepsilon \rightarrow 0$. To obtain a more precise estimate, we expand $3 L_{1}$ and $L_{2}$ in powers of $\varepsilon$. This yields

$$
3 L_{1}=\frac{3 \pi}{\sqrt{1+\sqrt{\alpha_{1}^{*}+\varepsilon}}}=\pi \sqrt{5}\left\{1-\frac{25}{144} \varepsilon+O\left(\varepsilon^{2}\right)\right\} \quad \text { as } \quad \varepsilon \rightarrow 0,
$$


and

$$
L_{2}=\frac{\pi}{\sqrt{1-\sqrt{\alpha_{1}^{*}+\varepsilon}}}=\pi \sqrt{5}\left\{1+\frac{25}{16} \varepsilon+O\left(\varepsilon^{2}\right)\right\} \quad \text { as } \quad \varepsilon \rightarrow 0 .
$$

Therefore

$$
L_{\text {gap }}(\varepsilon)=\frac{125}{72} \pi \sqrt{5} \varepsilon+O\left(\varepsilon^{2}\right) \quad \text { as } \quad \varepsilon \rightarrow 0 .
$$

Based on the natural balance of terms in (4.1) we rescale the variables according to

$$
(a, b) \mapsto \sqrt{\varepsilon}(a, b), \quad t \mapsto \frac{1}{\varepsilon} t
$$

Using this rescaling and expanding (4.1) to $O(\varepsilon)$ we obtain the leading order problem

$$
\left\{\begin{array}{l}
\dot{a}=g_{1}(a, b) \stackrel{\text { def }}{=} a\left\{\frac{10}{9}(1-\delta)-\frac{3}{2} a^{2}+\frac{3}{2} a b-3 b^{2}\right\}, \\
\dot{b}=g_{2}(a, b) \stackrel{\text { def }}{=} 10 \delta b+\frac{1}{2} a^{3}-3 a^{2} b-\frac{3}{2} b^{3} .
\end{array}\right.
$$

Any solution of the system (4.4) recovers a solution to $(4.1)$ to $O\left(\varepsilon^{2}\right)$.

4.1. Stationary solutions. The stationary solutions of the system (4.4) are the points where the null clines $\Gamma_{1}$ and $\Gamma_{2}$, defined by

$$
\Gamma_{i}=\left\{(a, b): g_{i}(a, b)=0\right\} \quad \text { for } \quad i=1,2,
$$

intersect. Plainly, $\Gamma_{1}$ consists of two components:

$$
\Gamma_{1}^{(1)}=\{(a, b): a=0\} \quad \text { and } \quad \Gamma_{1}^{(2)}=\left\{(a, b): \frac{3}{2} a^{2}-\frac{3}{2} a b+3 b^{2}=\frac{10}{9}(1-\delta)\right\} \text {, }
$$

and

$$
\Gamma_{2}=\left\{(a, b): \frac{1}{2} a^{3}-3 a^{2} b-\frac{3}{2} b^{3}+10 \delta b=0\right\} .
$$

Note that both $\Gamma_{1}$ and $\Gamma_{2}$ are invariant under the transformation $(a, b) \rightarrow(-a,-b)$, i.e., they are symmetric with respect to the origin. In what follows we shall at times only discuss the null clines in the first and the second quadrant, since the structure there will be duplicated in the third and the fourth quadrant.

We immediately see that the intersection of $\Gamma_{1}^{(1)}$ and $\Gamma_{2}$ consists of two points in the $(a, b)$-plane:

$$
O=(0,0) \quad \text { for } \delta \in \mathbf{R} \quad \text { and } \quad P=\left(0, \sqrt{\frac{20 \delta}{3}}\right) \quad \text { for } \delta \geq 0 .
$$

The first point yields the branch of trivial solutions and the second point yields the branch of stationary solutions of the system (4.4) which emanates from $3 L_{1}$ as described in [17] and [18]. It undergoes no bifurcation as $\alpha$ increases through $\alpha_{1}^{*}$.

It is the third branch, the one which emanates from $L_{2}$, for which $a$ is not identially zero, and which corresponds the the points of intersection of $\Gamma_{1}^{(2)}$ and $\Gamma_{2}$ that we now concentrate on. We see that for any $\delta<1$ the null cline $\Gamma_{1}^{(2)}$ is an ellipse in the $(a, b)$-plane around the origin, and that $\Gamma_{2}$ defines a continuous curve connecting 

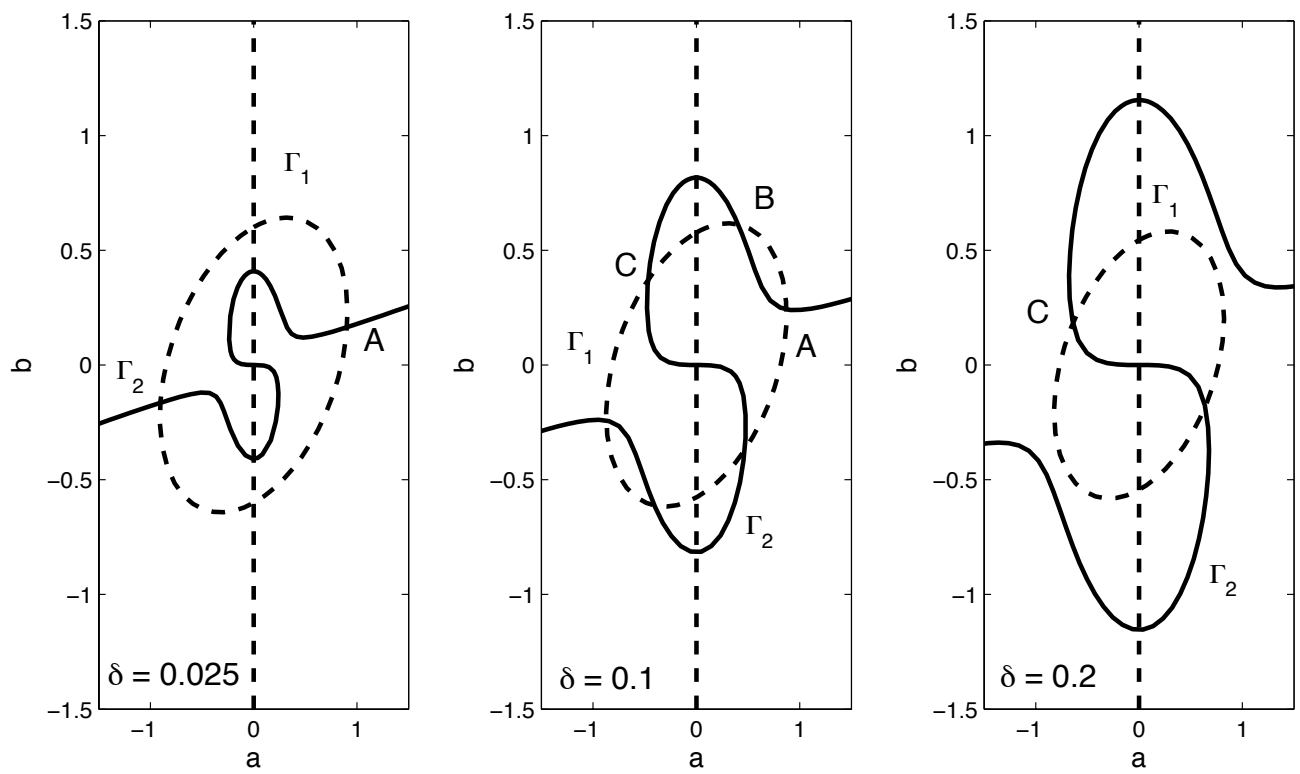

FiguRe 5. Plots of $\Gamma_{1}^{(2)}$ and $\Gamma_{2}$ for $\delta=0.025, \delta=0.1$ and $\delta=0.2$

$(0,0)$ to the point at infinity. Thus, for any $\delta<1$ there is always at least one stationary solution of the system (4.4). In Figure 5 we show the null clines $\Gamma_{1}^{(2)}$ and $\Gamma_{2}$ for $\delta=0.025$ (left), $\delta=0.1$ (middle) and $\delta=0.2$ (right)

The graphs of $\Gamma_{1}^{(2)}$ and $\Gamma_{2}$ suggest that there are three critical values of $\delta: \delta_{1}<$ $\delta_{2}<\delta_{3}$ in the interval $(0,1)$ such that

- if $0<\delta<\delta_{1}$, then $\Gamma_{1}^{(2)} \cap \Gamma_{2}$ consists of one point in the first quadrant, which we denote by $A(\delta)$ and one point, $-A(\delta)$ in the third quadrant.

When $\delta$ increases and passes through $\delta_{1}$, two additional points of intersection appear in the second quadrant, and two appear in the fourth quadrant:

- if $\delta_{1}<\delta<\delta_{2}$, then $\Gamma_{1}^{(2)} \cap \Gamma_{2}$ consists of two points in the second quadrant, which we denote by $B(\delta)$ and $C(\delta)$, and one point, $A(\delta)$, in the first quadrant. Symmetrically located with respect to the origin, there are points $-A(\delta),-B(\delta)$ and $-C(\delta)$ in the lower half plane.

When $\delta$ increases $B(\delta)$ moves to the $b$-axis and crosses it at $\delta_{2}$, so that

- if $\delta_{2}<\delta<\delta_{3}$, then $\Gamma_{1}^{(2)} \cap \Gamma_{2}$ consists of the origin, one point $C(\delta)$ in the second quadrant, and two points, $A(\delta)$ and $B(\delta)$ in the first quadrant. Symmetrically located with respect to the origin, there are points $-A(\delta),-B(\delta)$ and $-C(\delta)$ in the lower half plane.

When $\delta=\delta_{3}$ the points $A(\delta)$ and $B(\delta)$ coalesce to a point in the first quadrant and subsequently disappear, so that 


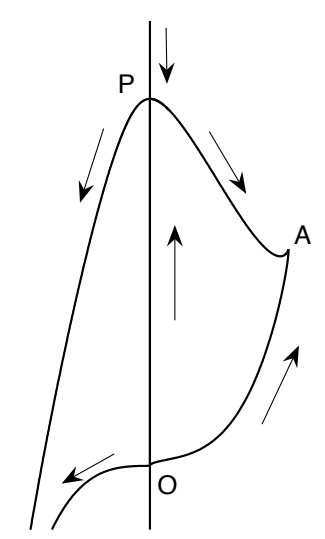

$\delta=0.025$

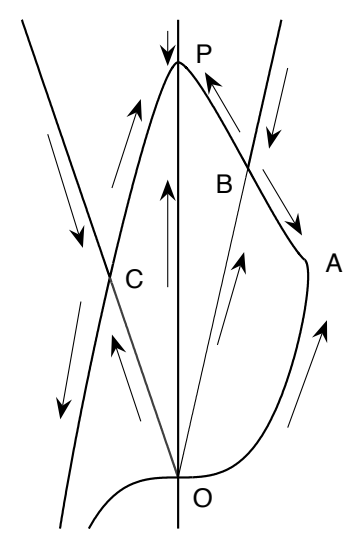

$\delta=0.1$

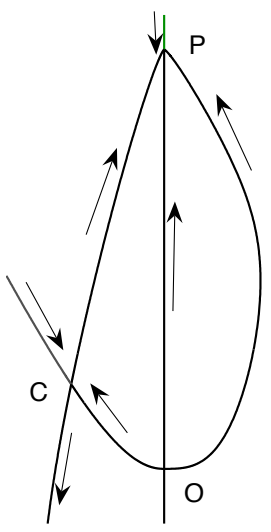

$\delta=0.2$

Figure 6. Connecting orbits in the phase plane when $\delta=0.025 \epsilon$ $\left(0, \delta_{1}\right), \delta=0.1 \in\left(\delta_{2}, \delta_{3}\right)$ and $\delta=0.2 \in\left(\delta_{3}, 1\right)$

- if $\delta_{3}<\delta<1$, then $\Gamma_{1}^{(2)} \cap \Gamma_{2}$ consists of one point, $C(\delta)$ in the second quadrant and one point, $-C(\delta)$, in the fourth quadrant.

Remark The points $B(\delta)$ and $C(\delta)$ move together as $\delta \rightarrow \delta_{1}^{+}$and the points $B(\delta)$ and $A(\delta)$ move together as $\delta \rightarrow \delta_{2}^{-}$.

In Appendix A we prove all these statements. There we find that

$$
\delta_{1}=0.050785 \ldots, \quad \delta_{2}=0.052631 \ldots \quad \text { and } \quad \delta_{3}=0.15191 \ldots
$$

4.2. Stability. The stability of the stationary solutions obtained above can easily be established by means of an analysis of the vector field of the system (4.4).

As a first observation, we note that $\Gamma_{1}^{(1)}=\{(a, b): a=0\}$ is an invariant set. This set contains three stationary points: the points $P$ and $-P$ as well as the origin $O$. Within this set, the origin is a repellor and the points $\pm P$ are both attractors. Thus, the origin is always unstable.

To discuss the nontrivial stationary solutions, we consider the three ranges of $\delta$-values: $\left(0, \delta_{1}\right),\left(\delta_{1}, \delta_{2}\right),\left(\delta_{2}, \delta_{3}\right)$ and $\left(\delta_{3}, 1\right)$ in succession. Graphs of orbits in the ranges $\left(0, \delta_{1}\right),\left(\delta_{2}, \delta_{3}\right)$ and $\left(\delta_{3}, 1\right)$ are shown in Figure 6.

The range $\mathbf{0}<\delta<\delta_{1}$ : We have four nontrivial stationary points: $\pm P$ and $\pm A$. By symmetry the characters of $\pm P$ and $\pm A$ are the same.

- Both $P$ and $-P$ are saddles: their stable manifold is $\Gamma_{1}^{(1)}$ and their unstable manifold is perpendicular to the $b$-axis. Therefore, both $P$ and $-P$ are unstable.

- The points $A$ and $-A$ are both stable nodes.

The range $\delta_{\mathbf{1}}<\delta<\delta_{\mathbf{2}}$ : We have four nontrivial stationary points: $P, A, B$ and $C$ in the upper half plane, and their symmetric images $-P,-A,-B$ and $-C$ in the lower half plane. 
- The points $\pm A$ and $\pm B$ are stable nodes.

- The points $\pm P$ and $\pm C$ are saddles, and hence unstable.

The range $\delta_{\mathbf{2}}<\delta<\delta_{\mathbf{3}}$ : We have nontrivial stationary points: $P, A, B$ and $C$ in the upper half plane and their symmetric images $-P,-A,-B$ and $-C$ in the lower half plane.

- The points $\pm P$ and $\pm A$ and their symmetric images are stable nodes .

- The points $B$ and $C$ and their symmetric images are saddles, and hence unstable.

The range $\delta_{\mathbf{3}}<\delta<1$ : We have four nontrivial stationary points: $\pm P$ and $\pm C$.

- The points $\pm P$ are stable nodes .

- The points $\pm C$ are saddles .

When we translate these results to the solution branches in the $(L, u)$-plane, we obtain the following description:

- The branch emanating from the point $\left(3 L_{1}, 0\right)$ starts at $\delta=0$ and corresponds to the point $P$ in the phase plane.

- For $0<\delta<\delta_{2}$ the branch is unstable.

- For $\delta_{2}<\delta<1$ it is stable.

- The branch emanating from the point $\left(L_{2}, 0\right)$ starts at $\delta=1$ and corresponds successively to the points $C, B$ and $A$ in the phase plane.

- For $\delta_{1}<\delta<1$ the branch corresponds to $C$ and it is unstable. At $\delta_{1}$ the branch bends back, and passes through a saddle-node bifurcation. This part of the branch corresponds to the point $B$ and is stable for $\delta_{1}<\delta<\delta_{2}$, i.e., until it intersects with the branch which emanates from $\left(3 L_{1}, 0\right)$. Continuing beyond $\delta_{2}$ it becomes unstable until at $\delta_{3}$ it bends back again through a saddle-node bifurcation. Then, for $0<\delta<\delta_{3}$ the branch corresponds to the point $A$ and it is stable again.

The stability properties of the different pieces of the branches are indicated in Figure 7 .

\section{Further BIFURCATiON POINTS}

In this section we study structures of bifurcation curves in the $(L, u)$-plane when higher order bifurcation points (nearly) coincide, i.e., when there exists an $\alpha^{*} \in(0,1)$ and positive integers $m$ and $n$ such that

$$
n L_{1}\left(\alpha^{*}\right)=m L_{2}\left(\alpha^{*}\right) .
$$

Since $L_{1}<L_{2}$ it follows that $m<n$. Throughout we only consider odd integers. it is readily verified that $(5.1)$ occurs when

$$
\alpha^{*}=\alpha_{m, n}^{*} \stackrel{\text { def }}{=}\left(\frac{n^{2}-m^{2}}{n^{2}+m^{2}}\right)^{2} .
$$




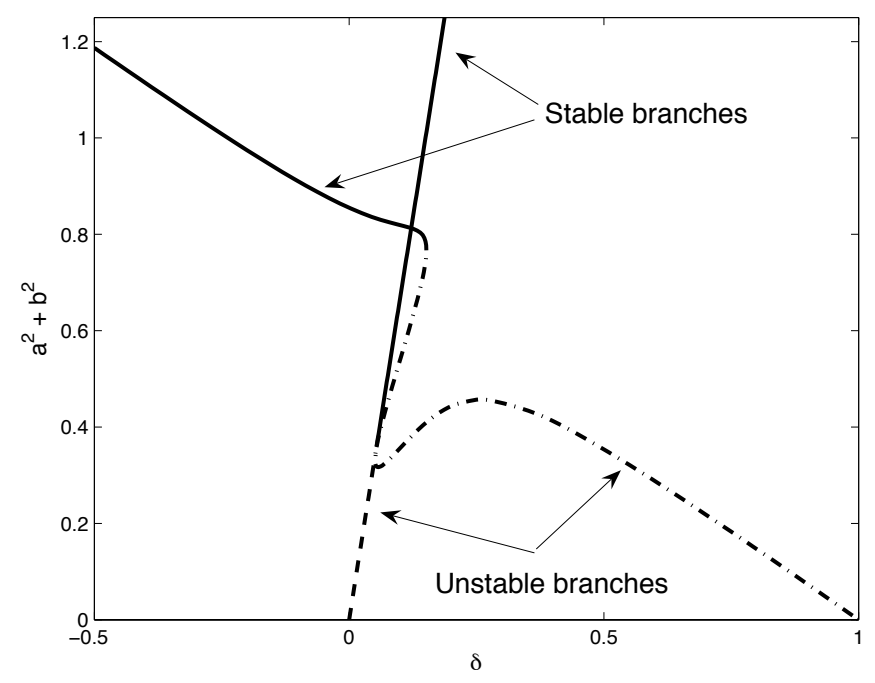

FiguRE 7. Stability of solution branches.

As a first observation we note that when $n=3 m$, then (5.1) becomes $3 L_{1}\left(\alpha^{*}\right)=$ $L_{2}\left(\alpha^{*}\right)$, so that $\alpha^{*}=\alpha_{1}^{*}$, and we return to the structure described in Section 4 . In Figure 8 , in which $5 L_{1} \approx 3 L_{2}$, we see a different type of structure. This type of connecting branch will be the main focus in this section.

Let us generalize the center manifold theorem, Theorem 2.3, from Section 2 to this situation.

Theorem 5.1. Suppose that (5.1) holds for some $\alpha^{*} \in(0,1)$ and for odd integers $m$ and $n$. Then, if $L=n L_{1}^{*}$,

(a) There exists a two-dimensional center manifold $X^{c}$ spanned by the eigenfunctions $\varphi_{m}$ and $\varphi_{n}$.

(b) If $n=m+2 \ell$ for $\ell \geq 1$, then the dimension of the unstable manifold $X^{u}$ is equal to $\ell-1$.

Proof. Since we have chosen $L$ such that $L=n L_{1}\left(\alpha^{*}\right)=m L_{2}\left(\alpha^{*}\right)$, it follows that

$$
\lambda_{m}(L)=0 \quad \text { and } \quad \lambda_{n}(L)=0 .
$$

Remembering that $\lambda_{k}(L)=P(k \pi / L)$ we therefore have

$$
P(\xi)=0 \quad \text { for } \quad \xi=\xi_{m} \quad \text { and } \quad \xi=\xi_{n} \quad \text { where } \quad \xi_{k}=\frac{k \pi}{L} .
$$

Given the properties of the function $P(\xi)$, we see immediately that

$$
P(\xi)>0 \quad \text { for } \quad \xi \in\left(0, \xi_{m}\right) \cup\left(\xi_{n}, \infty\right) .
$$

and

$$
P(\xi)<0 \quad \text { for } \quad \xi \in\left(\xi_{m}, \xi_{n}\right) \text {. }
$$




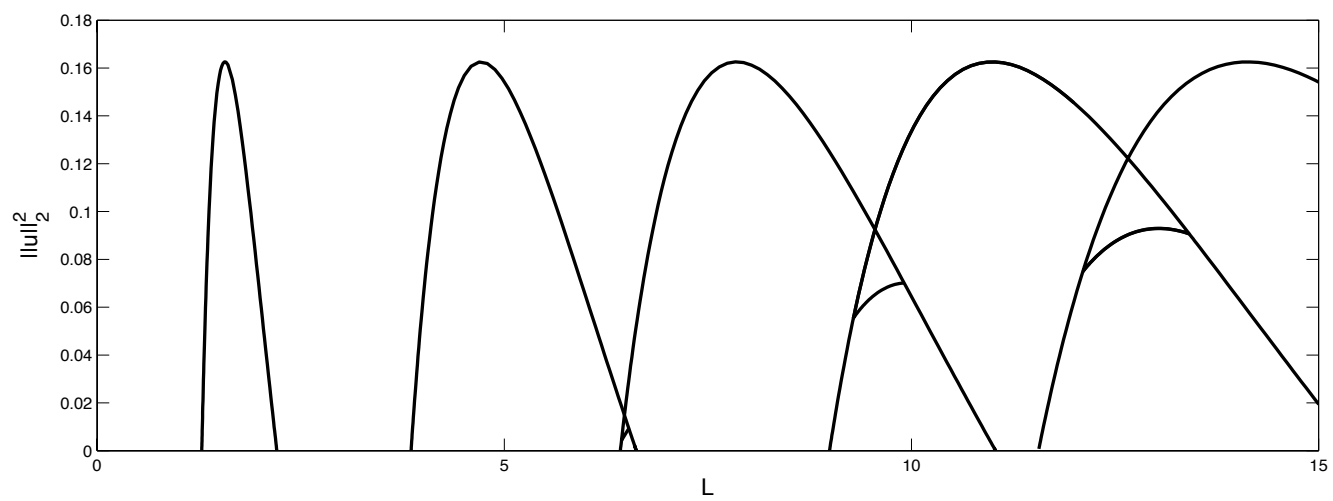

FiguRE 8. Global bifurcation diagram when $\alpha=0.24$ and $5 L_{1} \approx 3 L_{2}$

Since $\xi_{n}=\xi_{m}+(2 l \pi / L)$, this implies that

$$
\lambda_{k}(L)=P\left(\xi_{k}\right)<0 \quad \text { for } \quad k=1,2, \ldots, \ell-1,
$$

so that the dimension of the unstable manifold is equal to $\ell-1$.

Remark We note that the dimension of $X^{u}$ is positive, precisely when there is a bifurcation point inside an interval $\left(n L_{1}, n L_{2}\right)$. Thus while we can construct the local bifurcation diagram for any bifurcation point, only those which occur at $\alpha_{1}^{*}$ are related to stable dynamics.

We now extend the calculations of Section 3 to the general case. Projecting the Swift-Hohenberg equation onto the space $X^{c}=\operatorname{span}\left\{\varphi_{m}, \varphi_{n}\right\}$ and writing

$$
u(x, t)=a(t) \varphi_{m}(x)+b(t) \varphi_{n}(x),
$$

we obtain the system

$$
\left\{\begin{array}{l}
\dot{a}=-P\left(\frac{m \pi}{L}\right) a-\frac{3}{2} a^{3}-3 a b^{2}+\frac{3}{2} a^{2} b\left(\varphi_{3 m}, \varphi_{n}\right), \\
\dot{b}=-P\left(\frac{n \pi}{L}\right) b-3 a^{2} b-\frac{3}{2} b^{3}+\frac{1}{2} a^{3}\left(\varphi_{3 m}, \varphi_{n}\right),
\end{array}\right.
$$

where we have used the fact that

$$
\left(\varphi_{m}^{3}, \varphi_{n}\right)=-\frac{1}{2}\left(\varphi_{3 m}, \varphi_{n}\right) \quad \text { and } \quad\left(\varphi_{m}^{2}, \varphi_{n}^{2}\right)=1
$$

We note that when $3 m=n$ we retrieve the system (4.1) in Section 4 , and we obtain the same structure, albeit scaled by a factor $m$.

If $3 m \neq n$ the inner products in (5.3) vanish and we obtain the new system

$$
\left\{\begin{array}{l}
\dot{a}=-P\left(\frac{m \pi}{L}\right) a-\frac{3}{2} a^{3}-3 a b^{2} \\
\dot{b}=-P\left(\frac{n \pi}{L}\right) b-3 a^{2} b-\frac{3}{2} b^{3}
\end{array}\right.
$$


Next, we increase $\alpha$ by $\varepsilon$, i.e., we put $\alpha=\alpha_{m, n}^{*}+\varepsilon(\varepsilon>0)$. Then the bifurcation points $n L_{1}(\alpha)$ and $m L_{2}(\alpha)$ move apart. As in Section 4 we write

$$
L=L_{m, n}^{*}+\delta L_{\text {gap }}
$$

where now

$$
L_{m, n}^{*}=n L_{1}\left(\alpha^{*}\right)=\pi \sqrt{\frac{m^{2}+n^{2}}{2}} \quad \text { and } \quad L_{\text {gap }}=\frac{\pi}{8 \sqrt{2}} \frac{\left(n^{2}+m^{2}\right)^{7 / 2}}{n^{2} m^{2}\left(n^{2}-m^{2}\right)} \varepsilon+\mathcal{O}\left(\varepsilon^{2}\right),
$$

and

$$
L=L_{m, n}^{*}+\delta L_{\text {gap }}=L_{m, n}^{*}\left\{1+\frac{\varepsilon}{8} \frac{\left(n^{2}+m^{2}\right)^{2}}{n^{2}\left(n^{2}-m^{2}\right)}\left(\frac{m^{2}+n^{2}}{m^{2}} \delta-1\right)+\mathcal{O}\left(\varepsilon^{2}\right)\right\} .
$$

Rescaling the variables as in (4.3) and expanding $P\left(\frac{m \pi}{L}\right)$ and $P\left(\frac{n \pi}{L}\right)$ into powers of $\varepsilon$ as before then leads to the system

$$
\left\{\begin{array}{l}
\dot{a}=a\left\{\frac{n^{2}+m^{2}}{n^{2}}(1-\delta)-\frac{3}{2} a^{2}-3 b^{2}\right\}, \\
\dot{b}=b\left\{\frac{n^{2}+m^{2}}{m^{2}} \delta-3 a^{2}-\frac{3}{2} b^{2}\right\} .
\end{array}\right.
$$

5.1. Stationary solutions. The null clines of the system (5.3) are two ellipses, as well as the two axes:

$$
\Gamma_{1}=\Gamma_{1}^{(1)} \cup \Gamma_{1}^{(2)}, \quad \Gamma_{2}=\Gamma_{2}^{(1)} \cup \Gamma_{2}^{(2)}
$$

where

$$
\Gamma_{1}^{(1)}=\{(a, b): a=0\} \quad \text { and } \quad \Gamma_{1}^{(2)}=\left\{(a, b): a^{2}+2 b^{2}=\frac{2}{3} \frac{n^{2}+m^{2}}{n^{2}}(1-\delta)\right\}
$$

and

$$
\Gamma_{2}^{(1)}=\{(a, b): b=0\} \quad \text { and } \quad \Gamma_{2}^{(2)}=\left\{(a, b): 2 a^{2}+b^{2}=\frac{2}{3} \frac{n^{2}+m^{2}}{m^{2}} \delta\right\} .
$$

They are shown in Figure 9.

We see that for all $\delta \in(0,1)$ the set $\Gamma_{1} \cap \Gamma_{2}$ contains the origin as well as the points $P$ and $-P$ and $Q$ and $-Q$, where

$$
P(\delta)=\left(0, \sqrt{\frac{2\left(n^{2}+m^{2}\right)}{3 m^{2}} \delta}\right) \quad \text { and } \quad Q(\delta)=\left(\sqrt{\frac{2\left(n^{2}+m^{2}\right)}{3 n^{2}}(1-\delta), 0}\right) .
$$

The points $\pm P$ correspond to the branch which bifurcates at $n L_{1}$ and the points $\pm Q$ correspond to the branch which bifurcates at $m L_{2}$. These solutions are the only ones for $0<\delta<\delta_{1}$ and for $\delta_{2}<\delta<1$, where

$$
\delta_{1}=\frac{m^{2}}{m^{2}+2 n^{2}} \quad \text { and } \quad \delta_{2}=\frac{2 m^{2}}{2 m^{2}+n^{2}} .
$$

At $\delta_{1}$ and $\delta_{2}$ branches of solutions bifurcate from $\pm P$ and $\pm Q$ and for $\delta_{1}<\delta<\delta_{2}$ we have four additional solutions. We denote them by $\pm A$ and $\pm B$, where

$$
A(\delta)=K\left(\sqrt{\left(m^{2}+2 n^{2}\right) \delta-m^{2}}, \sqrt{2 m^{2}-\left(2 m^{2}+n^{2}\right) \delta}\right),
$$



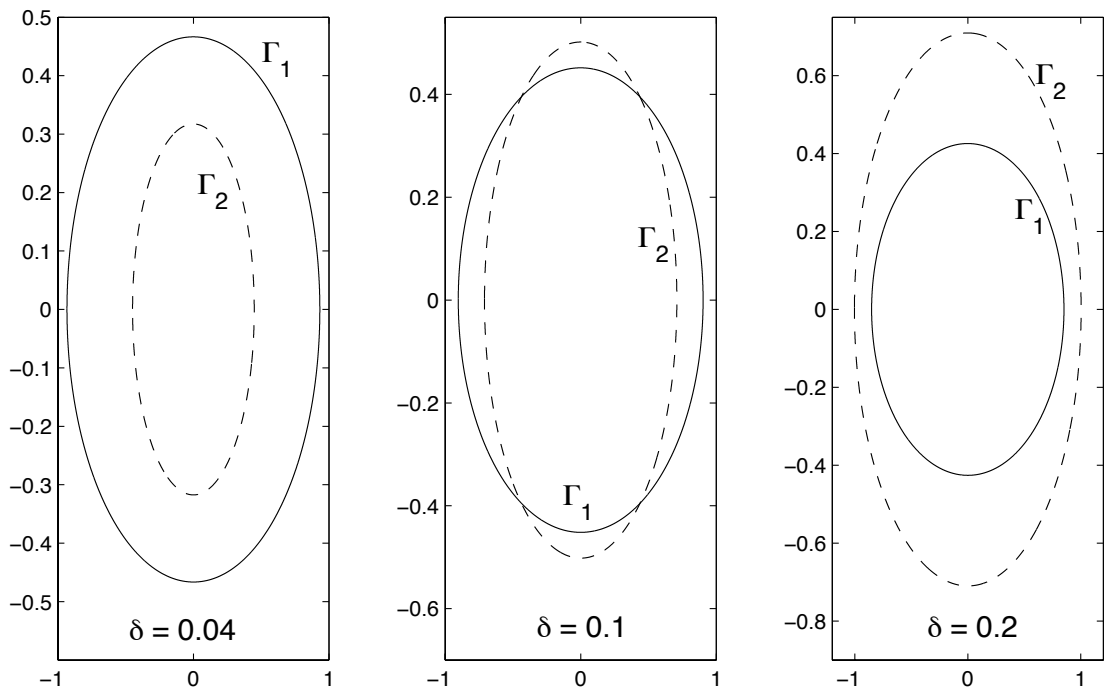

Figure 9. Plots of $\Gamma_{1}^{(2)}$ and $\Gamma_{2}^{(2)}$ for $\delta=0.04, \delta=0.1$ and $\delta=0.2$.

where $K=(1 /(3 m n)) \sqrt{2\left(m^{2}+n^{2}\right)}$, and

$$
B(\delta)=K\left(-\sqrt{\left(m^{2}+2 n^{2}\right) \delta-m^{2}}, \sqrt{2 m^{2}-\left(2 m^{2}+n^{2}\right) \delta}\right),
$$

5.2. Stability. A stability analysis of these solutions such as presented in Section 4 shows the followong:

If $0<\delta<\delta_{1}$, then

$-P$ and $-P$ are saddles and hence unstable.

$-Q$ and $-Q$ are stable nodes.

If $\delta_{1}<\delta<\delta_{2}$, then

$-P$ and $-P$, and $Q$ and $-Q$ are all stable nodes.

$-A$ and $-A$ and $B$ and $-B$ are saddles and unstable.

If $\delta_{2}<\delta<1$, then

$-P$ and $-P$ are stable nodes.

$-Q$ and $-Q$ are saddles and unstable.

Let us finally translate these results to the bifurcation picture in the $\left(L,\|u\|^{2}\right)$ diagram. For convenience we denote the branch which bifurcates at $n L_{1}$ by $\mathcal{C}_{1}$ and the branch which bifurcates at $m L_{2}$ by $\mathcal{C}_{2}$.

- The branch $\mathcal{C}_{1}$ corresponds to the point $P$; it is unstable for $0<\delta<\delta_{2}$ and stable for $\delta_{1}<\delta<1$.

- The branch $\mathcal{C}_{2}$ corresponds to the point $Q$; it is unstable for $\delta_{2}<\delta<1$ and stable for $0<\delta<\delta_{2}$. 

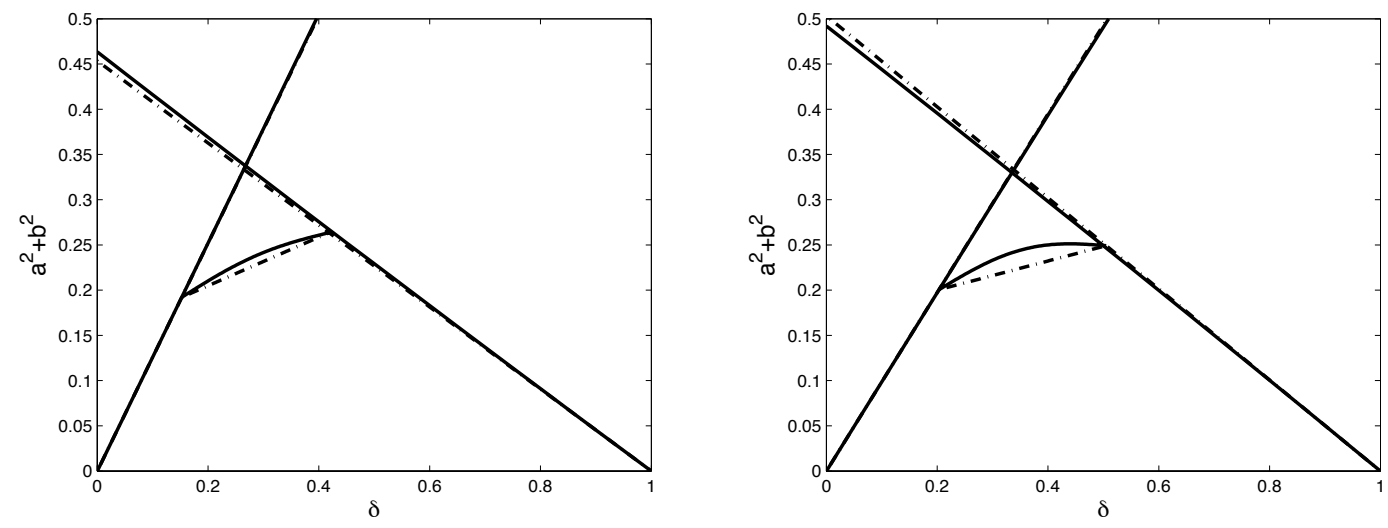

Figure 10. Comparison of the asymptotic and numerical bifurcation diagrams. Dashed lines are asymptotic solutions, solid are numerical. Left: $m=3$ and $n=5$. Right: $m=5$ and $n=7$.

- There are secondary bifurcations from $\mathcal{C}_{1}$ and $\mathcal{C}_{2}$ at, respectively, $\delta_{1}$ and $\delta_{2}$. These branch points are connected by a branch $\mathcal{C}_{3}$ which spans $\left(\delta_{1}, \delta_{2}\right)$. The branch $\mathcal{C}_{3}$ is unstable.

A comparison of these structures with numerical computations is presented in Figure 10. Note that

$$
\|u\|^{2}=a^{2}+b^{2}
$$

so that the branch $\mathcal{C}_{3}$ becomes

$$
\|u\|^{2}=\frac{2}{9} \frac{m^{2}+n^{2}}{m^{2} n^{2}}\left\{m^{2}+\left(n^{2}-m^{2}\right) \delta\right\}, \quad \delta_{1} \leq \delta \leq \delta_{2},
$$

i.e., $\mathcal{C}_{3}$ is a straight line in the $\left(L,\|u\|^{2}\right.$ )-plane (see Figure 10$)$.

\section{Global minimizers}

In Sections 4 and 5 we have computed solutions of Problem (1.3) in the center manifold $X^{c}$ of the trivial solutions at certain critical lengths, whenever $m L_{1} \approx n L_{2}$ and $m L_{1}<n L_{2}$ for some odd integers $m$ and $n$. The same analysis also yielded the stability properties of these solutions in $X^{c}$.

Since Problem (1.3) is a gradient system, it is interesting to know which of these solutions is the global minimizer. In this section we determine which of the solutions we obtained in $X^{c}$ is the global minimizer in $X^{c}$ given any value of $\delta \in[0,1]$. To this end we compute the value of the Lyapunov functional $V(a, b)$ along the solution branches in the two structures disussed in the previous sections, the one we find when $n=3 m$ and the one we find when $n \neq 3 m$. We discuss the two structures in succession. 

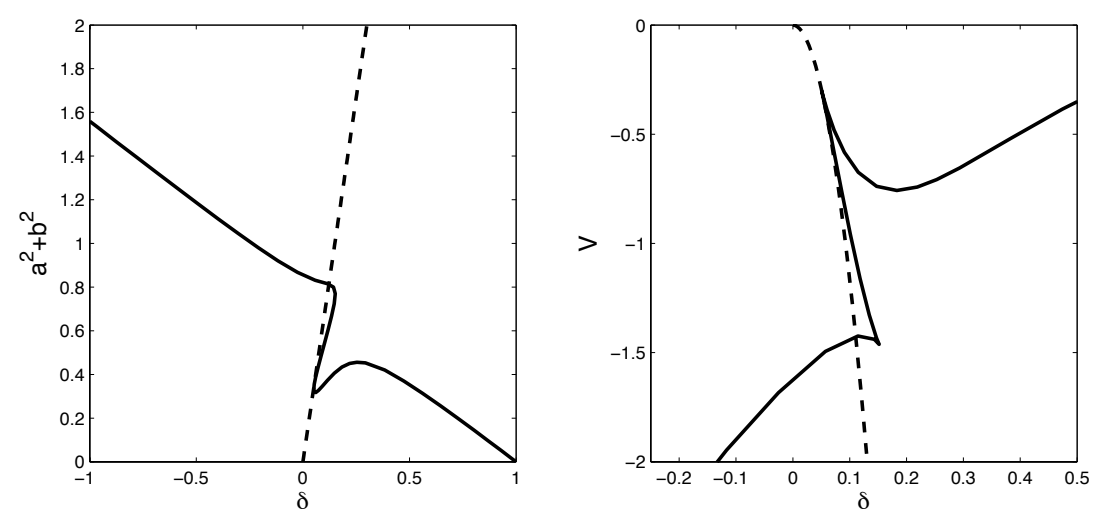

FiguRE 11. Solution branches (left) and scaled Lyapunov function $V$ along the branches (right) when $m=1$ and $n=3$

Case I: $\mathbf{n}=\mathbf{3 m}$. We recall from Section 4 that in this case the Lyapunov functional $V(a, b)$ associated with Problem (4.1) is given by

$$
V(a, b)=\frac{1}{2} P\left(\frac{\pi}{L}\right) a^{2}+\frac{1}{2} P\left(\frac{3 \pi}{L}\right) b^{2}+\frac{3}{8} a^{4}-\frac{1}{2} a^{3} b+\frac{3}{2} a^{2} b^{2}+\frac{3}{8} b^{4} .
$$

We put $\alpha=\alpha_{1}^{*}+\varepsilon$, carry out the scaling

$$
(a, b) \mapsto \sqrt{\varepsilon}(a, b), \quad t \rightarrow \frac{1}{\varepsilon} t \quad \text { and } \quad V(a, b) \rightarrow \frac{1}{\varepsilon} V(a, b),
$$

and expand $P\left(\frac{\pi}{L}\right)$ and $P\left(\frac{3 \pi}{L}\right)$. This yields for the scaled Lyapunov functional

$$
V(a, b)=-\frac{20}{9}(1-\delta) a^{2}-20 \delta b^{2}+\frac{3}{8} a^{4}-\frac{1}{2} a^{3} b+\frac{3}{2} a^{2} b^{2}+\frac{3}{8} b^{4} .
$$

In Figure 11 we show graphs of the Lyapunov functional $J(u ; L)$ (numerically computed) and of of $V(a, b)$ along the solution branches near $3 L_{1}$, when $3 L_{1} \approx L_{2}$.

Case II: $\mathbf{n} \neq \mathbf{3 m}$. Proceeding as in Case I, we find that the scaled Lyapunov function $V(a, b)$ is now given by

$$
V(a, b)=-\frac{n^{2}+m^{2}}{2 n^{2}}(1-\delta) a^{2}-\frac{n^{2}+m^{2}}{2 m^{2}} \delta b^{2}+\frac{3}{8} a^{4}+\frac{3}{2} a^{2} b^{2}+\frac{3}{8} b^{4} .
$$

In Figure 12 we show graphs of the Lyapunov functional $J(u ; L)$ (numerically computed) and of $V(a, b)$ along the solution branches near $5 L_{1}$, when $5 L_{1} \approx 3 L_{2}$.

\section{The quintic SwifT-Hohenberg Equation}

In this section we expand the analysis of the previous sections to the SwiftHohenberg equation in which the cubic term $u^{3}$ has been replaced by the quintic 

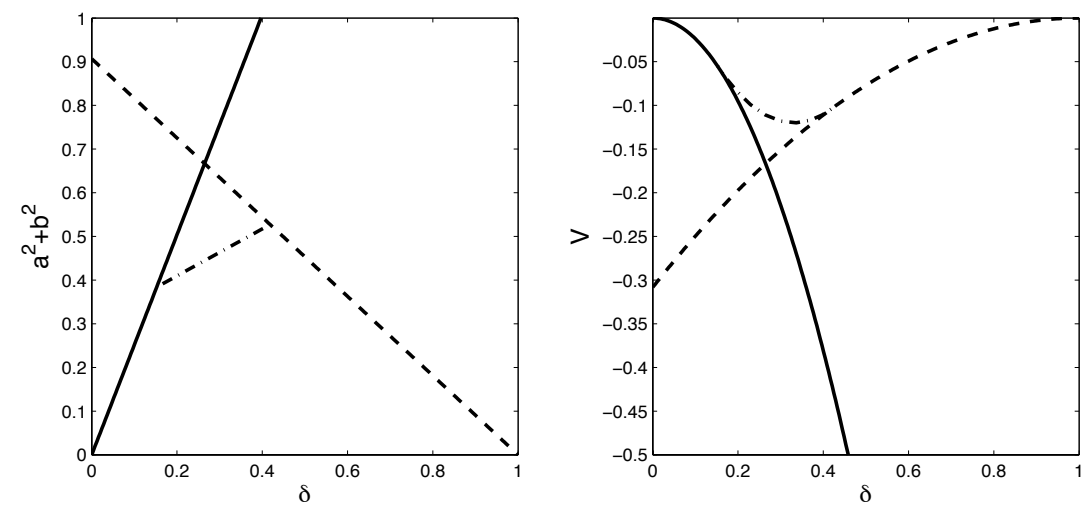

FiguRE 12. Solution branches (left) and scaled Lyapunov function $V$ along the branches (right) when $m=3$ and $n=5$

polynomial $-u^{3}+u^{5}$. Specifically, we consider the following initial-boundary value problem

$$
\left\{\begin{array}{lr}
u_{t}=-u_{x x x x}-2 u_{x x}-(1-\alpha) u+u^{3}-u^{5} & \text { for } 0<x<L, t>0 \\
u=0 \text { and } u_{x x}=0 & \text { at } x=0, L, t>0 \\
u(x, 0)=u_{0}(x) & \text { for } 0<x<L .
\end{array}\right.
$$

Like for the Swift-Hohenberg equation, the linearized version of Problem (7.1) is Problem (2.1) which has been studied in detail in Section 2. We find the same critical lengths $L_{1}$ and $L_{2}$ and the same eigenvalues $\lambda_{n}$ and eigenfunctions $\varphi_{n}$. Solution branches bifurcate from the points $(L, u)=\left(n L_{k}, 0\right)(k=1,2)$ for every $n \geq 1$. However, whereas in the Swift-Hohenberg equation, the bifurcations at $\left(n L_{1}, 0\right)$ are supercritical and subcritical at $\left(n L_{2}, 0\right)$, here they are subcritical at $\left(n L_{1}, 0\right)$ and supercritical and at $\left(n L_{2}, 0\right)$. Proceeding as in [18] we can find the local behavior at $\left(L_{1}, 0\right)$ and $\left(L_{2}, 0\right)$ :

Theorem 7.1. Let $\alpha>0$. There exists a unique branch in the $(L, u)$-plane of nontrivial solutions of Problem (7.1) which emanates from the trivial solution at $L_{1}$. Its local behavior is given by

$$
\|u\|^{2} \sim \frac{8}{3 \pi} \sqrt{\alpha}(1+\sqrt{\alpha})^{3 / 2}\left(L_{1}-L\right) \quad \text { as } \quad L \nearrow L_{1} .
$$

Theorem 7.2. Let $0<\alpha<1$. There exists a unique branch in the $(L, u)$-plane of nontrivial solutions of Problem (7.1) which emanates from the trivial solution at $L_{2}$. Its local behavior is given by

$$
\|u\|^{2} \sim \frac{8}{3 \pi} \sqrt{\alpha}(1-\sqrt{\alpha})^{3 / 2}\left(L_{2}-L\right) \quad \text { as } \quad L \searrow L_{2}
$$

In Figure 13 we show two global bifurcation diagrams, one for $\alpha=0.42$ and one for $\alpha=0.49$, in which these properties clearly show up. We also see the characteristic 

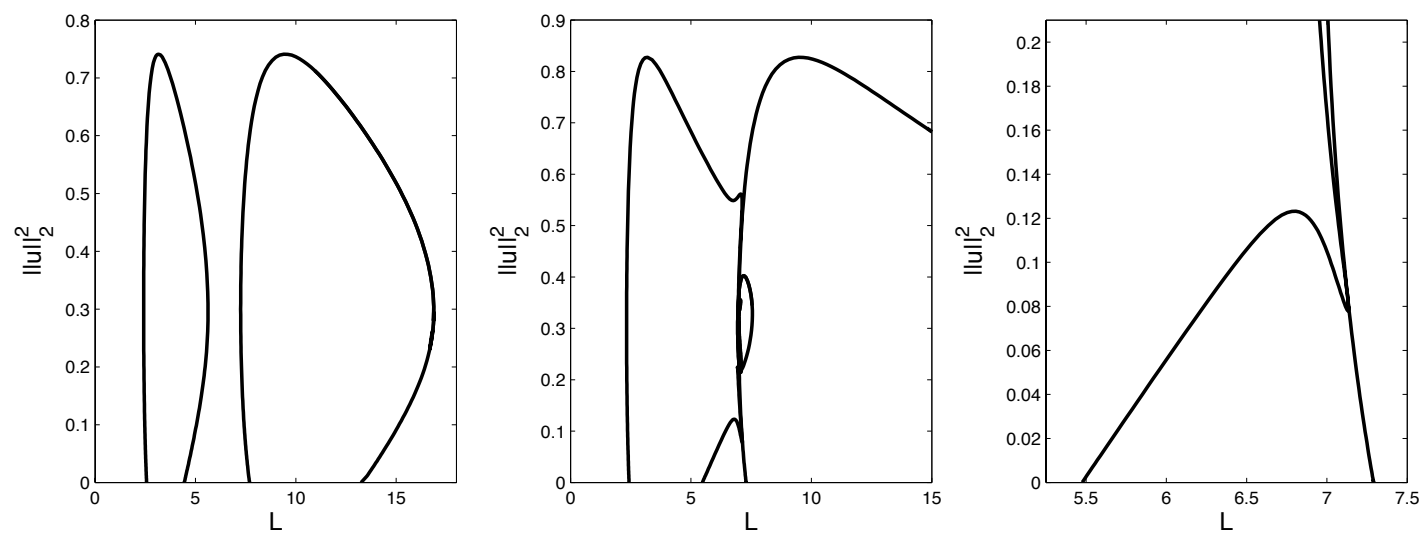

FiguRE 13. Sample bifurcation diagrams for de-stabilising nonlinearity. Notice that here the branches emanate from $L_{1}$ and $L_{2}$ in the opposite direction as in the stabilising case. Left: $\alpha=.42$ Middle: $\alpha=.49$. Right: Detail of $\alpha=.49$ showing the same local behavior as constructed in Section 4.

structure when $3 L 1 \approx L_{2}$, which we studied in Sections 4 and 6 . However, here it "flipped over".

Because the solution branches bifurcating from the points $\left(k L_{1}, 0\right)$ and $\left(k L_{2}, 0\right)$ now point in the opposite direction from the one in the stabilizing case, direction, we need to fix $\alpha<\alpha_{m, n}^{*}$ to ensure that $n L_{1}>m L_{2}$. Thus, in order to study the solution set when $3 L_{1} \approx L_{2}$, we write

$$
\alpha=\alpha_{1}^{*}-\varepsilon \quad \text { for } \quad \varepsilon>0,
$$

and put

$$
L=3 L_{1}+\delta L_{\text {gap }}, \quad \text { where } \quad L_{\text {gap }}=L_{2}-3 L_{1} .
$$

Note that now $L_{\text {gap }}<0$. Rescaling the equations as in Sections 4 and 5, we now obtain the $\mathcal{O}(\varepsilon)$ system

$$
\left\{\begin{array}{l}
\dot{a}=-a\left\{\frac{10}{9}(1-\delta)-\frac{3}{2} a^{2}+\frac{3}{2} a b-3 b^{2}\right\} \\
\dot{b}=-10 \delta b-\frac{1}{2} a^{3}+3 a^{2} b+\frac{3}{2} b^{3}
\end{array}\right.
$$

when $n=3 m$. Reversing time, and setting $t=-\tau$, we obtain the system (4.4) where the dots now denote differentiation with respect to $\tau$.

It is evident that for every $\delta \in[0,1]$, the family of stationary solutions is the same as that of (4.4), but, because $L$ now decreases when $\delta$ increases, the bifucation picture is the mirror image of the one in Section 4.

If $n \neq 3 m$, the analysis is similar to the one in Section 5 .

Finally, because all the nontrivial equilibrium solutions of (4.4) are either stable nodes or saddles, the equilibrium solutions of (7.2) are all unstable nodes or saddles, 
and therefore all unstable. It is easily seen that the origin $(a, b)=(0,0)$ is now a stable node.

\section{Appendix A. Additional algebraic Details}

In order to prove the results of Section 4 it will be convenient to express the null clines $\Gamma_{1}^{(2)}$ and $\Gamma_{2}$ in terms of polar coordinates, writing

$$
a=r \cos (\theta) \quad \text { and } \quad b=r \sin (\theta) .
$$

By symmetry this is possible if the ray

$$
R_{\tau}=\{(a, b): b=\tau a, \quad b>0\}, \quad \tau \in \mathbf{R}
$$

intersects each null cline at most at one point, and is never tangent to one of the null clines. This is clearly the case for $\Gamma_{2}$ which is an ellipse.

In fact, it is also the case for $\Gamma_{1}^{(2)}$. Suppose to the contrary that for some $\tau_{0}$ the ray $R_{\tau_{0}}$ is tangent to $\Gamma_{1}^{(2)}$ at the point $\left(a_{0}, b_{0}\right)$. Then, at $\left(a_{0}, b_{0}\right)$ we have (dropping the subscript "0"):

$$
a^{3}-6 a^{2} b-3 b^{3}=20 \delta b, \quad \text { and } \quad b=\tau a,
$$

so that

$$
a^{2}\left(1-6 \tau-3 t^{3}\right)=20 \delta \tau
$$

as well as

$$
3 a^{2}-12 a b-6 a^{2} \tau-9 b^{2} \tau=20 \delta \tau .
$$

Because $\Gamma_{1}^{(2)}$ and $R_{\tau}$ are tangent, we also have

$$
3 a^{2}\left(1-6 \tau-3 \tau^{3}\right)=20 \delta \tau
$$

It follows that $1-6 \tau-3 \tau^{3}=0$, so that $\tau=\tau^{*} \stackrel{\text { def }}{=} 0.16444 \ldots$. This would imply that $\delta=0$, a contradiction.

As a corollary we conclude by continuity that $R_{\tau}$ intersects $\Gamma_{1}^{(2)}$ at most at one point.

Thus, we can write $\Gamma_{1}^{(2)}$ as $r_{1}(\theta)$ for $\theta_{0}<\theta<\pi$, where $\theta_{0}$ will be determined later, and $\Gamma_{2}$ as $r_{2}(\theta)$ for $0<\theta<\pi$. Substituting (A.1) we obtain the following equations:

$$
\text { (A.4) } 20 \delta \sin (\theta)+r_{1}^{2} F(\theta)=0 \quad \text { and } \quad \frac{20}{27}(1-\delta)=r_{2}^{2} G(\theta), \quad 0<\theta<\pi,
$$

where

$$
F(\theta)=\cos ^{3}(\theta)-6 \cos ^{2}(\theta) \sin (\theta)-3 \sin ^{3}(\theta)
$$

and

$$
G(\theta)=\cos ^{2}(\theta)-\cos (\theta) \sin (\theta)+2 \sin ^{2}(\theta) .
$$

It follows from the equations in (A.4) that $\theta$ needs to be so restricted that $F(\theta)<0$ and $G(\theta)>0$. When we divide $F(\theta)$ by $\sin ^{3}(\theta)$, the first condition can be expressed as

$$
\cot ^{3}(\theta)-6 \cot ^{2}(\theta)-3<0 \quad \Longrightarrow \quad-\infty<\cot (\theta)<1 / \tau^{*}
$$


so that $\theta \in\left(\theta^{*}, \pi\right)$, where $\theta^{*}=\cot ^{-1}\left(1 / \tau^{*}\right)$. Because

$$
G(\theta)=\left(\cos (\theta)-\frac{1}{2} \sin (\theta)\right)^{2}+\frac{7}{4} \sin ^{2}(\theta)>0 \quad \text { for all } \quad \theta \in[0,2 \pi)
$$

the second condition poses no restriction on $\theta$.

Thus,

$$
r_{1}^{2}(\theta)=-20 \delta \frac{\sin (\theta)}{F(\theta)} \quad \text { and } \quad r_{2}^{2}(\theta)=\frac{20}{27}(1-\delta) \frac{1}{G(\theta)}
$$

At the points where the null clines intersect we have $r_{1}=r_{2}$, so that

$$
-20 \delta \frac{\sin (\theta)}{F(\theta)}=\frac{20}{27}(1-\delta) \frac{1}{G(\theta)},
$$

or

$$
\sin (\theta) G(\theta)+\gamma F(\theta)=0, \quad \gamma=\frac{1-\delta}{27 \delta} .
$$

If we divide by $\sin ^{3}(\theta)$ and write $x=\cot (\theta)$, we obtain the cubic polynomial equation

$$
H(x ; \gamma) \stackrel{\text { def }}{=} x^{2}-x+2+\gamma\left(x^{3}-6 x^{2}-3\right)=0, \quad-\infty<t<1 / \tau^{*} .
$$

Plainly,

$$
H^{\prime}(x ; \gamma)=2 x-1-\gamma\left(3 x^{2}-12 x\right) \quad \text { and } \quad H^{\prime \prime}(x ; \gamma)=2-\gamma(6 x-12) \text {. }
$$

Thus $H^{\prime}(x ; \gamma)=0$ if $x \in\left\{\xi_{-}, \xi_{+}\right\}$, where

$$
\xi_{ \pm}=\frac{1}{3 \gamma}\left(6 \gamma-1 \pm \sqrt{(1-6 \gamma)^{2}+3 \gamma}\right) \text {. }
$$

It is easily verified that

$$
H^{\prime \prime}\left(\xi_{-} ; \gamma\right)<0 \quad \text { and } \quad H^{\prime \prime}\left(\xi_{+} ; \gamma\right)>0
$$

Therefore equation (A.7) will have three zeros if $H\left(\xi_{+} ; \gamma\right)<0<H\left(\xi_{-} ; \gamma\right)$, two zeros if $H\left(\xi_{-} ; \gamma\right)=0$ or $H\left(\xi_{+} ; \gamma\right)=0$ and one zero if either $H\left(\xi_{-} ; \gamma\right)<0$ or $H\left(\xi_{+} ; \gamma\right)>0$. These conditions can easily be translated into conditions on $\gamma$. An elementary computation yields the values

$$
\gamma_{1}=0.69224 \ldots \quad \rightarrow \quad \delta_{1}=0.050785 \ldots
$$

and

$$
\gamma_{3}=0.206767 \quad \rightarrow \quad \delta_{3}=0.15191 \ldots,
$$

and that the null clines $\Gamma_{1}^{(2)}$ and $\Gamma_{2}$ intersect at 3 points when $\delta \in\left(\delta_{1}, \delta_{3}\right)$. At $\delta=\delta_{2}$, one of these critical point lies on the $b$-axis, i.e., $\theta=\pi / 2$. Then $x=\cot (\theta)=0$ and we deduce from equation (A.7) that $\gamma=2 / 3$ and hence $\delta_{2}=0.05263158 \ldots$. 


\section{APPENDix B. NumericAl APPROXIMATION}

In this paper we have used a continuation package for Matlab called MATCONT [12] for the computation of all bifurcation diagrams. This solves the algebraic system of equations corresponding to the finite difference approximation to the ODE problem. Continuation is then done via pseudo-arclength parametrization.

The fourth-derivative is approximated with a five point stencil and the second derivative with a three point stencil. Because of the symmetry properties of the solutions, the numerical problem was solved on $x \in[-L / 2, L / 2]$ with the boundary conditions

$$
u^{\prime}(-L / 2)=u^{\prime \prime \prime}(-L / 2)=0 \quad \text { and } \quad u^{\prime}(L / 2)=u^{\prime \prime \prime}(L / 2)=0 .
$$

Derivatives at the boundary are approximated with ghost points.

Because continuation is being done with respect to the interval length the spatial grid spacing changes along the branches. In all cases the number of grid points $N$ was chosen such that the grid spacing $h$ satisfied $h=L /\{2(N-1)\}<1 / 10$.

\section{REFERENCES}

[1] Berg, J.B. van den \& R.C.A.M. van der Vorst, Stable patterns for fourth order parabolic equations, Duke Math. J. 115 (2002) 513-558.

[2] Bodenschatz, E., W. Pesch \& G. Ahlers, Recent developments in Rayleigh-Bénard convection, Ann. Rev. Fluid Mech. 32 (2000) 709-778.

[3] Berg, G.J.B. van den, L.A. Peletier \& W.C. Troy, Global branches of multi-bump periodic solutions of the Swift-Hohenberg equation, Arch. Rational Mech. Anal. 158 (2001) 91-153.

[4] Caginalp, G. \& P.C. Fife, Higher order phase field models and detailed anisotropy, Phys. Rev. B 34 (1986) 4940-4943.

[5] Chaparova, J., Existence and numerical approximation of periodic solutions of semilinear fourth order differential equations, J. Math. Anal. Appl. 273 (2003) 121-136.

[6] Chaparova, J., L.A. Peletier \& S. Tersian, Existence and nonexistence of nontrivial solutions of semilinear sixth order ordinary differential equations, to appear in Appl. Math. Letters, 2003.

[7] Chaparova, J., L.A. Peletier \& S. Tersian, Existence and nonexistence of nontrivial solutions of semilinear fourth- and sixth-order ordinary differential equations, Adv. Diff. Eqns 8 (2003) 1237-1258.

[8] Collet, P. \& J.P. Eckmann, Instabilities and fronts in extended systems, Princeton Series in Physics, Princeton University Press, 1990.

[9] Cross, M.C. \& P.C. Hohenberg, Pattern formation outside of equilibrium, Rev. Mod. Phys. 65 (1993) 851-1112.

[10] Dee, G.T. \& W. van Saarloos, Bistable systems with propagating fronts leading to pattern formation, Phys. Rev. Lett. 60 (1988) 2641-2644.

[11] Gardner, R.A. \& C.K.R.T Jones, Traveling waves of a perturbed diffusion equation arising in a phase field model, Indiana Univ. Math. J., 38 (1989) 1197-1222.

[12] A. Ghooge, W. Govaerts \& Yu. Kuznetsov, MATCONT: A MATLAB package for numerical bifurcation analysis of ODEs, ACM Trans. Math. Soft. 26 2003, 141-163.

[13] Hale, J.K., Asymptotic behavior of dissipative systems, Mathematical Surveys and Monographs \#25, American Mathematical Society, Providence RI, 1988.

[14] Hohenberg, P.C. \& J.B. Swift, Effects of additive noise at the onset of Rayleigh-Bénard convection, Phys. Rev. A 46 (1992) 4773.

[15] Lega, J., J.V. Moloney \& A.C. Newell, Swift-Hohenberg equation for lasers, Phys. Rev. Lett. 73 (1994) 2978-2981. 
[16] Pomeau, Y. \& P. Manneville, Wave length selection in cellular flows, Phys. Letters 75A (1980) 296-298.

[17] Peletier, L.A. \& V. Rottschäfer, Large time behaviour of solutions of the Swift-Hohenberg equation, C. R. Acad. Sci. Paris, Ser I. 336 (2003) 225-230.

[18] Peletier, L.A. \& V. Rottschäfer, Pattern selection of solutions of the Swift-Hohenberg equation, Physica D 194 (2004) 95-126.

[19] Peletier, L.A. \& W.C. Troy, Spatial patterns: Higher order models in physics and mechanics, Birkhäuser, Boston, 2001.

[20] Sell, G.R., \& Y. You, Dynamics of evolutionary equations, Springer-Verlag, New York, 2002.

[21] Swift, J.B. \& P.C. Hohenberg, Hydrodynamic fluctuations at the convective instability, Phys. Rev. A 15 (1977), 319-328.

[22] Zimmerman, W., Propagating fronts near a Lifschitz point, Phys. Rev. Lett. 66 (1991) 1546.

Mathematical Institute, Leiden University, PB 9512, 2300 RA, Leiden, NL \& Centrum voor Wiskunde en Informatica, Postbus 94079, 1090 GB, Amsterdam, NL

E-mail address: peletier@math.leidenuniv.nl

Department of Mathematics, Simon Fraser University 8888 University Drive, Burnaby, British Columbia V5A 1S6 Canada

E-mail address: jfw@math.sfu.ca 